\title{
Wormhole Geometry and Noether Symmetry in $f(R)$ Gravity
}

\author{
M. Sharif ${ }^{* \dagger}$ and Iqra Nawazish $\ddagger$ \\ Department of Mathematics, University of the Punjab, \\ Quaid-e-Azam Campus, Lahore-54590, Pakistan.
}

\begin{abstract}
This paper investigates the geometry of static traversable wormhole through Noether symmetry approach in $f(R)$ gravity. We take perfect fluid distribution and formulate symmetry generators with associated conserved quantities corresponding to general form, powerlaw and exponential $f(R)$ models. In each case, we evaluate wormhole solutions using constant and variable red-shift functions. We analyze the behavior of shape function, viability of constructed $f(R)$ model and stability of wormhole solutions graphically. The physical existence of wormhole solutions can be examined through null/weak energy conditions of perfect fluid and null energy condition of the effective energy-momentum tensor. The graphical interpretation of constructed wormhole solutions ensures the existence of physically viable and traversable wormholes for all models. It is concluded that the constructed wormholes are found to be stable in most of the cases.
\end{abstract}

Keywords: Noether symmetry; Wormhole solution; $f(R)$ gravity. PACS: 04.20.Jb; 04.50.Kd; 95.36.+x.

*msharif.math@pu.edu.pk

${ }^{\dagger}$ Fellow, Pakistan Academy of Sciences, 3 Constitution Avenue, G-5/2, Islamabad.

†iqranawazish07@gmail.com 


\section{Introduction}

On the landscape of theoretical and observational modern cosmology, the most revolutionizing fact is believed to be the current cosmic accelerated expansion. Recent experiments indicate that this expansion must be due to some enigmatic force with astonishing anti-gravitational effects, known as dark energy. There are many proposals to explain its ambiguous nature. The $f(R)$ gravity is one of such proposals established by replacing geometric part of the Einstein-Hilbert action with this generic function depending on the Ricci scalar $R$. The fourth order non-linear field equations of this gravity keep triggering researchers to evaluate exact solution.

The study of exact solutions under assorted scenarios is extensively used to explore different cosmic aspects that unveil sophisticated picture of cosmic evolution. Sharif and Shmair[1] constructed vacuum as well as non-vacuum exact solutions of Bianchi I and $\mathrm{V}$ universe models in $f(R)$ gravity and also investigated physical behavior of these solutions. Gutiérrez-Piñeres and López-Monsalvo [2] evaluated exact vacuum solution for static axially symmetric spacetime in the same gravity and found that solution corresponds to naked singularity. Sharif and Zubair [3] considered interaction of matter with geometry to formulate some exact solutions of Bianchi I model. Gao and Shen [4] found a new method to formulate exact solutions of static spherically symmetric metric. They also analyzed some general properties of solutions like event horizon, singularity and deficit angle in Jordan and Einstein frames.

Noether symmetry approach is considered to be the most appreciable technique which explores not only exact solutions but also evaluates conserved quantities relative to symmetry generators associated with dynamical system. Capozziello et al. [5] formulated exact solution of static spherically symmetric metric for $f(R)$ power-law model. The same authors [6] generalized this work for non-static spherically symmetric spacetime and also discussed possible solutions for axially symmetric model. Vakili [7] studied the scalar field scenario of flat FRW model through this approach and discussed current cosmic phase via effective equation of state parameter corresponding

to quintessence phase. Momeni et al. [8] investigated the existence of Noether symmetry for isotropic universe model in mimetic $f(R)$ as well as $f(R, T)$ gravity theories ( $T$ denotes trace of energy-momentum tensor). Sharif and his collaborators [9] investigated cosmic evolution as well as current cosmic expansion through Noether symmetry approach. 
Our universe always bring eye opening questions for cosmologists regrading its surprising and mysterious nature. The existence of hypothetical geometries is considered as the most debatable issue which leads to wormhole geometry. A wormhole (WH) structure is defined through a hypothetical bridge or tunnel which allows a smooth connection among different regions only if there exists exotic matter (matter with negative energy density). The existence of a physically viable WH is questioned due to the presence of enough amount of exotic matter. Consequently, there is only one way to have a realistic WH model, i.e., the presence of exotic matter must be minimized. Besides the existence of such astrophysical configurations, the most crucial problem is stability analysis which defines their behavior against perturbations as well as enhances physical characterization. A singularity-free configuration identifies a stable state which successfully prevents the WH to collapse while a WH can also exist for quite a long time even if it is unstable due to very slow decay. The evolution of unstable system can lead to many phenomena of interest from structure formation to supernova explosions. To explore WH existence, different approaches have been proposed such as modified theories of gravity, non-minimal curvature-matter coupling, scalar field models etc [10].

The study of WH solutions has been of great interest in modified theories of gravity. Lobo and Oliveira [11] considered constant shape function and different fluids to explore WH solution in $f(R)$ gravity. Jamil et al. [12] formulated viable WH solutions for $f(R)$ power-law model and also considered particular shape function in the background of non-commutative geometry. Bahamonde et al. [13] constructed cosmological WH threaded by perfect fluid approaching to FRW universe in the same gravity. Mazharimousavi and Halilsoy [14 found a near-throat WH solution of $f(R)$ model admitting polynomial expansion and also satisfying necessary WH conditions for both vacuum as well as non-vacuum cases. Sharif and Fatima [15] discussed static spherically symmetric WH in galactic halo region as well as investigated non-static conformal WH in $f(\mathcal{G})$ gravity, ( $\mathcal{G}$ represents Gauss-Bonnet term). Noether symmetry approach elegantly explores the WH geometry by formulating exact solutions. Bahamonde et al. [16] obtained exact solutions of red-shift as well as shape functions through this approach and analyzed their geometric behavior graphically in scalar-tensor theory incorporating non-minimal coupling with torsion scalar.

In this paper, we study WH geometry threaded by perfect fluid via Noether symmetry approach in $f(R)$ gravity. The format of the paper is 
as follows. Section 2 explores basic review of $f(R)$ gravity. In section $\mathbf{3}$, we construct point-like Lagrangian which is used in section 4 to evaluate WH solutions for both constant as well as variable red-shift functions. Section $\mathbf{5}$ investigates stability of the constructed WH solutions. In the last section, we present final remarks.

\section{Basics of $f(R)$ Gravity}

We consider a minimally coupled action of $f(R)$ gravity given by

$$
\mathcal{I}=\int d^{4} x \sqrt{-g}\left[\frac{f(R)}{2 \kappa^{2}}+\mathcal{L}_{m}\right]
$$

where $g$ identifies determinant of the metric tensor $g_{\mu \nu}, f(R)$ describes a coupling-free function while $\mathcal{L}_{m}$ denotes Lagrangian density of matter. The metric variation of action (11) leads to

$$
f_{R} R_{\mu \nu}-\frac{1}{2} f g_{\mu \nu}-\nabla_{\mu} \nabla_{\nu} f_{R}+g_{\mu \nu} \square f_{R}=\kappa^{2} T_{\mu \nu}^{(m)}, \quad T_{\mu \nu}^{(m)}=g_{\mu \nu} \mathcal{L}_{m}-2 \frac{\partial \mathcal{L}_{m}}{\partial g^{\mu \nu}} .
$$

Here, $f_{R}$ shows the derivative of generic function $f$ with respect to $R, \nabla_{\mu}$ represents covariant derivative, $\square=\nabla_{\mu} \nabla^{\mu}$ and $T_{\mu \nu}^{(m)}$ denotes energy-momentum tensor. The equivalent form of Eq.(2) is

$$
G_{\mu \nu}=\frac{1}{f_{R}}\left(T_{\mu \nu}^{(m)}+T_{\mu \nu}^{(c)}\right)=T_{\mu \nu}^{e f f},
$$

where $G_{\mu \nu}, T_{\mu \nu}^{(c)}$ and $T_{\mu \nu}^{e f f}$ identify Einstein, curvature and effective energymomentum tensors, respectively. The curvature terms relative to generic function define $T_{\mu \nu}^{(c)}$ as

$$
T_{\mu \nu}^{(c)}=\frac{f-R f_{R}}{2} g_{\mu \nu}+\nabla_{\mu} \nabla_{\nu} f_{R}-\square f_{R} g_{\mu \nu} .
$$

The energy-momentum tensor corresponding to perfect fluid is

$$
T_{\mu \nu}^{(m)}=\left(\rho_{m}(r)+p_{m}(r)\right) u_{\mu} u_{\nu}+p_{m}(r) g_{\mu \nu},
$$

where $\rho_{m}$ and $p_{m}$ characterize energy density and pressure, respectively whereas $u_{\mu}$ denotes four velocity of the fluid as $u_{\mu}=\left(-e^{\frac{a(r)}{2}}, 0,0,0\right)$. 
The static spherically symmetric spacetime is [17]

$$
d s^{2}=-e^{a(r)} d t^{2}+e^{b(r)} d r^{2}+M(r)\left(d \theta^{2}+\sin ^{2} \theta d \phi^{2}\right),
$$

where $a, b$ and $M$ are arbitrary functions depending on radial coordinate $r$. The geodesic deviation equation determines that $M(r)=r^{2}, \sin r, \sinh r$ for $\mathcal{K}=0,1,-1$ ( $\mathcal{K}$ denotes curvature parameter) under the limiting behavior $M(r) \rightarrow 0$ as $r \rightarrow 0$, respectively [18. In case of $M(r)=r^{2}$, the spherical symmetry defines Morris-Thorne WH where $a(r)$ is recognized as red-shift function identifying gravitational red-shift while $e^{b(r)}$ explores the geometry of WH for $e^{b}=\left(1-\frac{h(r)}{r}\right)^{-1}, h(r)$ is known as shape function. In order to locate throat of a WH, radial coordinate must follow non-monotonic behavior such that it decreases from maximum to minimum value $r_{0}$ identifying $\mathrm{WH}$ throat at $h\left(r_{0}\right)=r_{0}$ and then it starts increasing from $r_{0}$ to infinity. To have a WH solution at throat, the condition $h^{\prime}\left(r_{0}\right)<1$ is imposed, where prime denotes derivative with respect to $r$. The flaring-out condition is the fundamental property of WH which demands $\frac{h(r)-h(r)^{\prime} r}{h(r)^{2}}>0$. For the existence of traversable WH, the surface should be free from horizons, the red-shift function must be finite everywhere and $1-h(r) / r>0$. To formulate the field equations for the action (1), we choose $\mathcal{L}_{m}=p_{m}(r)$ [19] and use Eqs.(2)-(15), it follows that

$$
\begin{aligned}
& \frac{e^{a}}{4 e^{b} M^{2}}\left(-4 M^{\prime \prime} M+2 b^{\prime} M^{\prime} M+M^{\prime 2}+4 M e^{b}\right)=\frac{1}{f_{R}}\left[\frac{e^{-b}\left(R f_{R}-f\right)}{2}\right. \\
& \left.-f_{R}^{\prime}\left(\frac{a^{\prime} e^{a}}{2 e^{b}}\right)+e^{a-b} f_{R}^{\prime \prime}+e^{a-b} f_{R}^{\prime}\left(\frac{a^{\prime}-b^{\prime}}{2}+\frac{M^{\prime}}{M}\right)+e^{a} \rho_{m}\right] \\
& -\frac{1}{4 M^{2}}\left(M^{\prime 2}+2 a^{\prime} M^{\prime} M-4 M e^{b}\right)=\frac{1}{f_{R}}\left[\frac{\left(f-R f_{R}\right)}{2}-\frac{b^{\prime} f_{R}^{\prime}}{2}-f_{R}^{\prime}\right. \\
& \left.\times e^{a-b} f_{R}^{\prime}\left(\frac{a^{\prime}-b^{\prime}}{2}+\frac{M^{\prime}}{M}\right)\left(\frac{a^{\prime}-b^{\prime}}{2}+\frac{M^{\prime}}{M}\right)+e^{b} p_{m}\right] \\
& \frac{1}{4 M e^{b}}\left(M^{\prime} M\left(a^{\prime}-b^{\prime}\right)+2 M^{\prime \prime} M+M^{2} a^{\prime 2}-M^{2} a^{\prime} b^{\prime}-M^{\prime 2}+2 M^{2} a^{\prime \prime}\right) \\
& =\frac{1}{f_{R}}\left[M p_{m}+\frac{M^{\prime} f_{R}^{\prime}}{2 e^{b} M}+\frac{M\left(R f_{R}-f\right)}{2}-\frac{f_{R}^{\prime \prime}}{M e^{b}}-\frac{f_{R}^{\prime}}{M e^{b}}\left(\frac{a^{\prime}-b^{\prime}}{2}+\frac{M^{\prime}}{M}\right)\right] .
\end{aligned}
$$

The energy conditions provide a significant way to analyze physical existence of some cosmological geometries. For WH geometry, the violation of 
these conditions ensures the existence of a realistic WH. To define energy conditions, Raychaudhari equations are considered to be the most fundamental ingredients given as

$$
\begin{aligned}
& \frac{d \theta}{d \tau}=-\frac{1}{3} \theta^{2}-\sigma_{\mu \nu} \sigma^{\mu \nu}+\Theta_{\mu \nu} \Theta^{\mu \nu}-R_{\mu \nu} l^{\mu} l^{\nu}, \\
& \frac{d \theta}{d \tau}=-\frac{1}{2} \theta^{2}-\sigma_{\mu \nu} \sigma^{\mu \nu}+\Theta_{\mu \nu} \Theta^{\mu \nu}-R_{\mu \nu} k^{\mu} k^{\nu},
\end{aligned}
$$

where $\theta, l^{\mu}, k^{\mu}, \sigma$ and $\Theta$ represent expansion scalar, timelike vector, null vector, shear and rotation tensors. The first equation is defined for timelike congruence while the second is for null congruence. The positivity of the last term of both equations demands attractive gravity. For the EinsteinHilbert action, these conditions split into null (NEC) $\left(\rho_{m}+p_{m} \geq 0\right)$, weak (WEC) $\left(\rho_{m} \geq 0, \rho_{m}+p_{m} \geq 0\right)$, strong (SEC) $\left(\rho_{m}+p_{m} \geq 0, \rho_{m}+3 p_{m} \geq\right.$ $0)$ and dominant (DEC) $\left(\rho_{m} \geq 0, \rho_{m} \pm p_{m} \geq 0\right)$ energy conditions [20]. As the Raychaudhari equations are found to be purely geometric implying that $T_{\mu \nu}^{(m)} k^{\mu} k^{\nu} \geq 0$ can be replaced with $T_{\mu \nu}^{e f f} k^{\mu} k^{\nu} \geq 0$. Thus, the energy conditions in $f(R)$ gravity turn out to be [21]

$$
\begin{aligned}
\text { NEC : } & \rho_{\text {eff }}+p_{\text {eff }} \geq 0, \\
\text { WEC : } & \rho_{\text {eff }} \geq 0, \quad \rho_{\text {eff }}+p_{\text {eff }} \geq 0, \\
\text { SEC : } & \rho_{\text {eff }}+p_{\text {eff }} \geq 0, \quad \rho_{\text {eff }}+3 p_{\text {eff }} \geq 0, \\
\text { DEC : } & \rho_{\text {eff }} \geq 0, \quad \rho_{\text {eff }} \pm p_{\text {eff }} \geq 0 .
\end{aligned}
$$

Solving Eqs.(6) and (17), we obtain

$$
\begin{aligned}
p_{m} & =-\frac{f}{2}+e^{-b} f_{R}^{\prime}\left(\frac{a^{\prime}}{2}+\frac{M^{\prime}}{M}\right)-\frac{f_{R}}{4 e^{b} M^{2}}\left(2 M^{\prime 2}-4 M^{\prime \prime} M-a^{\prime 2} M^{2}\right. \\
& \left.+a^{\prime} b^{\prime} M^{2}+2 b^{\prime} M^{\prime} M-2 M^{2} a^{\prime \prime}\right) \\
\rho_{m} & =\frac{f_{R}}{4 e^{b} M^{2}}\left(M^{2} a^{\prime 2}-M^{2} a^{\prime} b^{\prime}+2 a^{\prime} M^{\prime} M+2 M^{2} a^{\prime \prime}\right)+e^{-b} f_{R}^{\prime \prime}+e^{-b} f_{R}^{\prime} \\
& \times\left(\frac{-b^{\prime}}{2}+\frac{M^{\prime}}{M}\right)+\frac{f}{2} .
\end{aligned}
$$

In $f(R)$ gravity, NEC relative to the effective energy-momentum tensor for (5) yields

$$
\rho_{e f f}+p_{e f f}=\frac{1}{2 e^{b}}\left(\frac{M^{\prime 2}}{M^{2}}+\frac{a^{\prime} M^{\prime}}{M}+\frac{b^{\prime} M^{\prime}}{M}-\frac{2 M^{\prime \prime}}{M}\right) .
$$




\section{Point-like Lagrangian}

In this section, we construct point-like Lagrangian corresponding to the action (11) via Lagrange multiplier approach. In this regard, we consider following form of gravitational action [22]

$$
\mathcal{I}=\int \sqrt{-g}[f(R)-\lambda(R-\bar{R})] d r
$$

where

$$
\begin{aligned}
\sqrt{-g} & =e^{\frac{a}{2}} e^{\frac{b}{2}} M, \quad \lambda=f_{R} \\
\bar{R} & =\frac{1}{e^{b}}\left(-\frac{a^{\prime 2}}{2}+\frac{a^{\prime} b^{\prime}}{2}-\frac{a^{\prime} M^{\prime}}{M}-\frac{2 M^{\prime \prime}}{M}+\frac{b^{\prime} M^{\prime}}{M}+\frac{M^{\prime 2}}{2 M^{2}}-a^{\prime \prime}+\frac{2 e^{b}}{M}\right)
\end{aligned}
$$

The dynamical constraint $\lambda$ is obtained by varying the action (13) with respect to $R$. In order to determine $p_{m}$, we consider Bianchi identity $\left(\nabla_{\mu} T^{\mu \nu}\right)$ whose radial component gives

$$
\frac{d p_{m}}{d r}+\frac{a^{\prime}(r)}{2}\left(p_{m}+\rho_{m}\right)=0
$$

Solving this differential equation with $p_{m}=\omega \rho_{m}$, it follows that

$$
\rho_{m}=\rho_{0} a^{-\frac{(1+\omega)}{2 \omega}}, \quad p_{m}=\omega \rho_{m}=\omega \rho_{0} a^{-\frac{(1+\omega)}{2 \omega}},
$$

where $\omega$ represents equation of state parameter. Inserting Eq.(14) and (16) in (13), we obtain

$$
\begin{aligned}
\mathcal{I} & =\int e^{\frac{a-b}{2}} M\left[f(R)-R f_{R}+\frac{f_{R}}{e^{b}}\left(-\frac{a^{\prime 2}}{2}+\frac{a^{\prime} b^{\prime}}{2}-\frac{a^{\prime} M^{\prime}}{M}-\frac{2 M^{\prime \prime}}{M}+\frac{b^{\prime} M^{\prime}}{M}\right.\right. \\
& \left.\left.+\frac{M^{\prime 2}}{2 M^{2}}-a^{\prime \prime}+\frac{2 e^{b}}{M}\right)+\omega \rho_{0} a^{-\frac{(1+\omega)}{2 \omega}}\right] d r .
\end{aligned}
$$

Eliminating second order derivatives via integration by parts from the above action and following Lagrangian density definition, we obtain point-like Lagrangian as

$$
\mathcal{L}\left(r, a, b, M, R, a^{\prime}, b^{\prime}, M^{\prime}, R^{\prime}\right)=e^{\frac{a}{2}} e^{\frac{b}{2}} M\left(f-R f_{R}+\omega \rho_{0} a^{-\frac{(1+\omega)}{2 \omega}}+\frac{2 f_{R}}{M}\right)
$$




$$
+\frac{e^{\frac{a}{2}} M}{e^{\frac{b}{2}}}\left\{f_{R}\left(\frac{M^{\prime 2}}{2 M^{2}}+\frac{a^{\prime} M^{\prime}}{M}\right)+f_{R R}\left(a^{\prime} R^{\prime}+\frac{2 M^{\prime} R^{\prime}}{M}\right)\right\} .
$$

For static spherically symmetric spacetime, the Euler-Lagrange equation and Hamiltonian of the dynamical system or energy function associated with point-like Lagrangian are defined as

$$
\frac{\partial \mathcal{L}}{\partial q^{i}}-\frac{d p_{i}}{d r}=0, \quad \mathcal{H}=\sum_{i} q^{i} p_{i}-\mathcal{L},
$$

where $q^{i}$ are generalized coordinates and $p_{i}=\frac{\partial \mathcal{L}}{\partial q^{\prime 2}}$ represents conjugate momenta. The variation of Lagrangian with respect to configuration space leads to

$$
\begin{aligned}
& e^{b}\left(f-R f_{R}+\omega \rho_{0} a^{-\frac{(1+\omega)}{2 \omega}}-(1+\omega) \rho_{0} a^{-\frac{(1+3 \omega)}{2 \omega}}+\frac{2 f_{R}}{M}\right)+\left(\frac{M^{\prime 2}}{2 M^{2}}+\frac{b^{\prime} M^{\prime}}{M}\right. \\
& \left.-\frac{2 M^{\prime \prime}}{M}\right) f_{R}+f_{R R}\left(b^{\prime} R^{\prime}-2 R^{\prime \prime}-\frac{2 M^{\prime} R^{\prime}}{M}\right)-2 R^{\prime 2} f_{R R R}=0 \\
& e^{b}\left(f-R f_{R}+\omega \rho_{0} a^{-\frac{(1+\omega)}{2 \omega}}+\frac{2 f_{R}}{M}\right)-f_{R}\left(\frac{M^{\prime 2}}{2 M^{2}}+\frac{a^{\prime} M^{\prime}}{M}\right)-f_{R R}\left(a^{\prime} R^{\prime}\right. \\
& \left.+\frac{2 M^{\prime} R^{\prime}}{M}\right)=0, \\
& e^{b}\left(f-R f_{R}+\omega \rho_{0} a^{-\frac{(1+\omega)}{2 \omega}}+\frac{2 f_{R}}{M}\right)+f_{R}\left(-\frac{a^{\prime 2}}{2}+\frac{a^{\prime} b^{\prime}}{2}-\frac{a^{\prime} M^{\prime}}{2 M^{\prime}}-\frac{M^{\prime \prime}}{M}-a^{\prime \prime}\right. \\
& \left.+\frac{b^{\prime} M^{\prime}}{2 M}+\frac{M^{\prime 2}}{2 M^{2}}\right)+f_{R R}\left(b^{\prime} R^{\prime}-a^{\prime} R^{\prime}-2 R^{\prime \prime}-\frac{M^{\prime} R^{\prime}}{M}\right)-2 R^{\prime 2} f_{R R R}=0 \\
& {\left[e^{b}\left(\frac{2}{M}-R\right)-\frac{a^{\prime 2}}{2}+\frac{a^{\prime} b^{\prime}}{2}-\frac{a^{\prime} M^{\prime}}{M}-\frac{2 M^{\prime \prime}}{M}+\frac{b^{\prime} M^{\prime}}{M}+\frac{M^{\prime 2}}{2 M^{2}}-a^{\prime \prime}\right] f_{R R}=0 .}
\end{aligned}
$$

The energy function and variation of Lagrangian relative to shape function yield

$$
e^{b}=\frac{\frac{f_{R} M^{\prime}}{M}\left(\frac{M^{\prime}}{2 M^{2}}+a^{\prime} M^{\prime}\right)+R^{\prime} f_{R R}\left(a^{\prime} M+2 M^{\prime}\right)}{f-R f_{R}+\omega \rho_{0} a^{-\frac{(1+\omega)}{2 \omega}}+\frac{2 f_{R}}{M}} .
$$

\section{Noether Symmetry Approach}

The physical characteristics of a dynamical system can be identified by constructing the associated Lagrangian which successfully describes energy content and the existence of possible symmetries of the system. In this regard, 
Noether symmetry approach provides an interesting way to construct new cosmological models and geometries in modified theories of gravity. According to well-known Noether theorem, group generator yields associated conserved quantity if point-like Lagrangian remains invariant under a continuous group. In order to investigate the presence of Noether symmetry and relative conserved quantity of static spherically symmetric metric, we consider a vector field [23]

$$
K=\tau\left(r, q^{i}\right) \frac{\partial}{\partial r}+\zeta^{i}\left(r, q^{i}\right) \frac{\partial}{\partial q^{i}},
$$

where $r$ behaves as an affine parameter while $\tau$ and $\zeta^{i}$ are unknown coefficients of the vector field $K$.

The presence of Noether symmetry is assured only if point-like Lagrangian satisfies the invariance condition and the vector field is found to be unique on tangent space. Consequently, the vector field acts as a symmetry generator generating associated conserved quantity. In this case, the invariance condition is defined as

$$
K^{[1]} \mathcal{L}+(D \tau) \mathcal{L}=D B\left(r, q^{i}\right),
$$

where $B$ denotes boundary term of the extended symmetry, $K^{[1]}$ describes first order prolongation and $D$ represents total derivative given by

$$
K^{[1]}=K+\left(D \zeta^{i}-q^{i} D \tau\right) \frac{\partial}{\partial q^{\prime}}, \quad D=\frac{\partial}{\partial r}+q^{i} \frac{\partial}{\partial q^{i}} .
$$

Noether symmetries coming from invariance condition (21) lead to identify associated conserved quantities through first integral. If the Lagrangian remains invariant under translation in time and position, then the first integral identifies energy and linear momentum conservation while rotationally symmetric Lagrangian yields conservation of angular momentum [24]. For invariance condition (21), the first integral is defined as

$$
\Sigma=B-\tau \mathcal{L}-\left(\zeta^{i}-q^{\prime i} \tau\right) \frac{\partial \mathcal{L}}{\partial{q^{\prime}}^{i}}
$$

For configuration space $Q=\{a, b, M, R\}$, the vector field $K$ and first order prolongation $K^{[1]}$ take the following form

$$
K=\tau \frac{\partial}{\partial r}+\alpha \frac{\partial}{\partial a}+\beta \frac{\partial}{\partial b}+\gamma \frac{\partial}{\partial M}+\delta \frac{\partial}{\partial R}, \quad K^{[1]}=\tau \frac{\partial}{\partial r}+\alpha \frac{\partial}{\partial a}+\beta \frac{\partial}{\partial b}
$$




$$
+\gamma \frac{\partial}{\partial M}+\delta \frac{\partial}{\partial R}+\alpha^{\prime} \frac{\partial}{\partial a^{\prime}}+\beta^{\prime} \frac{\partial}{\partial b^{\prime}}+\gamma^{\prime} \frac{\partial}{\partial M^{\prime}}+\delta^{\prime} \frac{\partial}{\partial R^{\prime}}
$$

where the radial derivative of unknown coefficients of vector field are defined as

$$
\sigma_{j}^{\prime}=D \sigma_{j}-q^{i} D \tau, \quad j=1 \ldots 4 .
$$

Here $\sigma_{1}, \sigma_{2}, \sigma_{3}$ and $\sigma_{4}$ correspond to $\alpha, \beta, \gamma$ and $\delta$, respectively. Inserting Eqs.(18), (24) and (25) in (21) and comparing the coefficients of $a^{\prime 2}, a^{\prime} b^{\prime} M^{\prime}, a^{\prime} M^{\prime 2}$ and $a^{\prime} R^{\prime 2}$, we obtain

$$
\tau,{ }_{a} f_{R}=0, \quad \tau,_{b} f_{R}=0, \quad \tau,_{M} f_{R}=0, \quad \tau,_{R} f_{R R}=0 .
$$

This equation implies that either $f_{R}=0$ or vice verse. The first choice leads to trivial solution. Therefore, we consider $f_{R} \neq 0$ and compare the remaining coefficients which yield the following system of equations

$$
\begin{aligned}
& B,_{b}=0, \quad \tau,_{a}=0, \quad \tau,_{b}=0, \quad \tau,_{M}=0, \quad \tau,_{R}=0, \\
& e^{\frac{a}{2}}\left(\gamma,{ }_{r} f_{R}+M \delta,_{r} f_{R R}\right)=e^{\frac{b}{2}} B,{ }_{a}, \\
& e^{\frac{a}{2}}\left(\alpha,{ }_{r} M+2 \gamma,{ }_{r}\right) f_{R R}=e^{\frac{b}{2}} B,_{R}, \\
& e^{\frac{a}{2}}\left(\alpha,{ }_{r} f_{R}+\gamma,{ }_{r} M^{-1} f_{R}+2 \delta,_{r} f_{R R}\right)=e^{\frac{b}{2}} B,{ }_{M}, \\
& \gamma,{ }_{a} f_{R}+M \delta,{ }_{a} f_{R R}=0, \\
& \gamma,{ }_{a} f_{R}+M \delta,{ }_{a} f_{R R}=0, \\
& \alpha,_{b} f_{R}+\gamma,_{b} M^{-1} f_{R}+2 \delta,_{b} f_{R R}=0, \\
& M \alpha{ }_{{ }_{b}} f_{R R}+2 \gamma,{ }_{b} f_{R R}=0 \text {, } \\
& M \alpha,_{R} f_{R R}+2 \gamma,_{R} f_{R R}=0 \text {, } \\
& \left.f_{R}\left(\alpha-\beta-2 \gamma M^{-1}+4 M \alpha,_{M}+4 \gamma,_{M}-2 \tau,_{r}\right)+f_{R R}\left(2 \delta+8 M \delta,_{M}\right)=\emptyset 36\right) \\
& f_{R}\left(\alpha-\beta+2 \alpha,_{a}-2 \tau,,_{r}+2 \gamma,{ }_{M}+2 \gamma,{ }_{a}\right)+f_{R R}\left(2 \delta+2 M \delta,_{M}+4 \delta,_{a}\right)=0(37) \\
& f_{R}\left(\alpha,_{R}+\gamma,_{R} M^{-1}\right)+f_{R R}\left(\alpha-\beta+M \alpha,_{M}+2 \gamma,_{M}-2 \tau,_{r}+2 \delta,_{R}\right)+2 \delta \\
& \times f_{R R R}=0 \text {, } \\
& 2 \gamma,_{R} f_{R}+f_{R R}\left(M \alpha-M \beta+2 \gamma+2 M \alpha,_{a}-2 M \tau,_{r}+4 \gamma,_{a}+2 M \delta,_{R}\right)+2 M \\
& \times \delta f_{R R R}=0 \text {, } \\
& e^{\frac{a}{2}} e^{\frac{b}{2}} M\left\{\frac{1}{2}\left(f-R f_{R}+\omega \rho_{0} a^{-\frac{(1+\omega)}{2 \omega}}+\frac{2 f_{R}}{M}\right)\left(\alpha+\beta+\tau,,_{r}\right)-\frac{1}{2} \alpha(1+\omega) \rho_{0}\right. \\
& \left.\times a^{-\frac{(1+3 \omega)}{2 \omega}}+\delta M\left(2 M^{-1}-R\right) f_{R R}\right\}+e^{\frac{a}{2}} e^{-\frac{b}{2}} \gamma\left(f-R f_{R}+\omega \rho_{0} a^{-\frac{(1+\omega)}{2 \omega}}\right)
\end{aligned}
$$




$$
=B,_{r} .
$$

In order to solve this system, we consider $M(r)=r^{2}$ and taking $B,_{a}, B,_{M}, B,_{R}=$ 0, Eqs.(27)-(35) give

$$
\alpha=Y_{2}(a, r), \quad \gamma=Y_{1}(r), \quad \delta=Y_{3}(r, R) .
$$

Inserting these values in Eqs.(36)-(39), we obtain

$$
Y_{1}(r)=0, \quad Y_{2}(a, r)=c_{2}, \quad Y_{3}(r, R)=\frac{c_{1} f_{R}}{f_{R R}}, \quad \beta=2 c_{1}+c_{2}-2 \tau_{, r},
$$

where $c_{1}$ and $c_{2}$ are arbitrary constants. For these solutions, the coefficients of symmetry generator turn out to be

$$
\alpha=c_{2}, \quad \beta=2 c_{1}+c_{2}, \quad \gamma=0, \quad \delta=\frac{c_{1} f_{R}}{f_{R R}}, \quad \tau=c_{0} .
$$

Substituting these coefficients in Eq.(40), we formulate boundary term and explicit form of $f(R)$ as follows

$$
\begin{aligned}
f(R) & =-\frac{1}{2\left(c_{1}+c_{2}\right)}\left[-(1+\omega) \rho_{0} a^{-\frac{(1+3 \omega)}{2 \omega}}+2 \omega\left(c_{1}+c_{2}\right) \rho_{0} a^{-\frac{(1+\omega)}{2 \omega}}\right. \\
& \left.-6 c_{4} e^{\frac{-a-b}{2}}\right], \quad B=c_{3}+c_{4} r^{3} .
\end{aligned}
$$

The coefficients of symmetry generator, boundary term and solution of $f(R)$ satisfy the system of Eqs.(27)-(39) for $c_{1}=0$. Thus, the symmetry generator and the corresponding first integral take the form

$$
\begin{aligned}
K & =c_{0} \frac{\partial}{\partial r}+c_{2} \frac{\partial}{\partial a}+c_{2} \frac{\partial}{\partial b}, \\
\Sigma & =c_{3}+c_{4} r^{3}-c_{0}\left[e^{\frac{a}{2}} e^{\frac{b}{2}} r^{2}\left(f-R f_{R}+\omega \rho_{0} a^{-\frac{(1+\omega)}{2 \omega}}+2 f_{R} r^{-2}\right)\right. \\
& \left.+\frac{e^{\frac{a}{2}} r^{2}}{e^{\frac{b}{2}}}\left\{f_{R}\left(2 r^{-2}+2 a^{\prime} r^{-1}\right)+f_{R R}\left(a^{\prime} R^{\prime}+4 R^{\prime} r^{-1}\right)\right\}\right] \\
& -c_{2} e^{\frac{a-b}{2}}\left(R^{\prime} r^{2} f_{R R}+2 r f_{R}\right) .
\end{aligned}
$$

The verification of Eq.(40) yields

$$
b(r)=\int \frac{8 c_{6} r^{2}+a^{\prime \prime} r^{2}+4 a^{\prime} r^{\prime}+a^{\prime 2} r^{2}-4 c_{7}}{r\left(4+a^{\prime} r\right)} d r+c_{5},
$$


where $c_{i}$ 's $(i=3 \ldots 8)$ are arbitrary constants and this solution satisfies Eq.(40) for $\omega=1,1 / 3,-1 / 3,-1$. To discuss physical features and geometry of WH via shape function, we take red-shift function, $a(r)=k$ and $a(r)=-\frac{k}{r}, k>$ 0 , where $k$ denotes constant [25]. In the following, we solve integral for both choices of red-shift function.

Case I: $a(r)=k$

We first consider red-shift function to be constant and evaluate $b(r)$ such as

$$
b(r)=c_{6} r^{2}-c_{7} \ln r+c_{5}
$$

Consequently, the shape function turns out to be

$$
h(r)=r\left(1-e^{-b(r)}\right)=r\left(1-c_{7} r e^{-c_{6} r^{2}-c_{5}}\right) .
$$

In this case, the explicit form of $f(R)$ reduces to

$f(R)=-\frac{1}{2 c_{2}}\left[-(1+\omega) \rho_{0} k^{-\frac{(1+3 \omega)}{2 \omega}}+2 \omega c_{2} \rho_{0} k^{-\frac{(1+\omega)}{2 \omega}}-6 c_{4} \sqrt{c_{7} r} e^{\frac{-c_{6} r^{2}-c_{5}-k}{2}}\right]$.

The $f(R)$ theory of gravity is one of the competitive candidates in modified theories of gravity as it naturally unifies two expansion phases of the universe, i.e., inflation at early times and cosmic acceleration at current epoch. The higher derivative of curvature terms with positive power are dominant at the early universe leading to the inflationary stage. The terms with negative power of the curvature serve as gravitational alternative for the dark energy that acts as a possible source to speed-up cosmic expansion [26]. Despite the fact that the ghost-free $f(R)$ theory is very interesting and useful as it passes solar system tests, it also suffers from instabilities. For instance, the theory with $\frac{1}{R}$ may develop the instability [27] whereas by adding a term of $R^{2}$ to this specific form of $f(R)$ model, one can easily eliminate this instability [28]. Therefore, the viable $f(R)$ models require to satisfy the following stability constraints $f_{R}(R)>0, f_{R R}(R)>0, R>R_{0}$ where $R_{0}$ is the current Ricci scalar 29].

In Figure 1, both plots indicate that the constructed $f(R)$ model (45) preserves the stability conditions. Figure $\mathbf{2}$ shows the graphical analysis of shape function. The upper left plot represents positive behavior of $h(r)$ while 

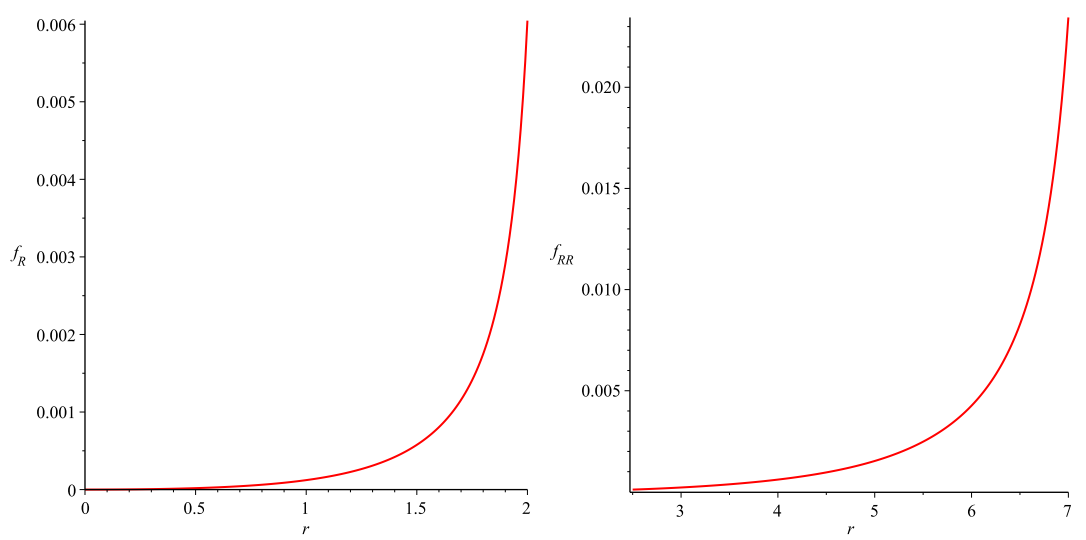

Figure 1: Plots of stability conditions of $f(R)$ model versus $r$ for $c_{2}=5$, $c_{4}=0.01, c_{5}=-0.35, c_{6}=0.1, c_{7}=-0.25, \rho_{0}=1$ and $k=0.5$.

the upper right indicates that the shape function admits asymptotic behavior. The lower left plot locates the WH throat at $r_{0}=4.4$ and the corresponding right plot identifies that $\frac{d h\left(r_{0}\right)}{d r}=0.9427<1$. To discuss physical existence of $\mathrm{WH}$, we insert constant red-shift function and Eq.(43) in (12) yielding

$$
\rho_{e f f}+p_{e f f}=\frac{r h^{\prime}(r)-h(r)}{r^{3}}<0,
$$

which satisfies the flaring-out condition. Consequently, NEC violates in this case, $\rho_{\text {eff }}+p_{\text {eff }}<0$ and assures the presence of repulsive gravity leading to traversable WH. In order to study the realistic existence of traversable WH, we analyze the behavior of NEC and WEC in Figure 3. Both plots indicate that energy density and pressure recover energy bounds as $\rho_{m} \geq 0$ and $\rho_{m}+p_{m} \geq 0$ implying physically acceptable traversable WH.

case II: $a(r)=-k / r$

In this case, we choose red-shift function in terms of $r$ leading to

$$
\begin{aligned}
a(r) & =-\frac{k}{r}, \quad b(r)=\frac{1}{8 r}\left(4 c_{6} r^{2}(2 r-k)-32 c_{8} r \ln r+\left(32 r-8 c_{7} r+c_{6} k r^{2}\right)\right. \\
& \left.\times \ln (4 r+k)-8 k / c_{8}\right)+c_{5}, \quad k>0 .
\end{aligned}
$$



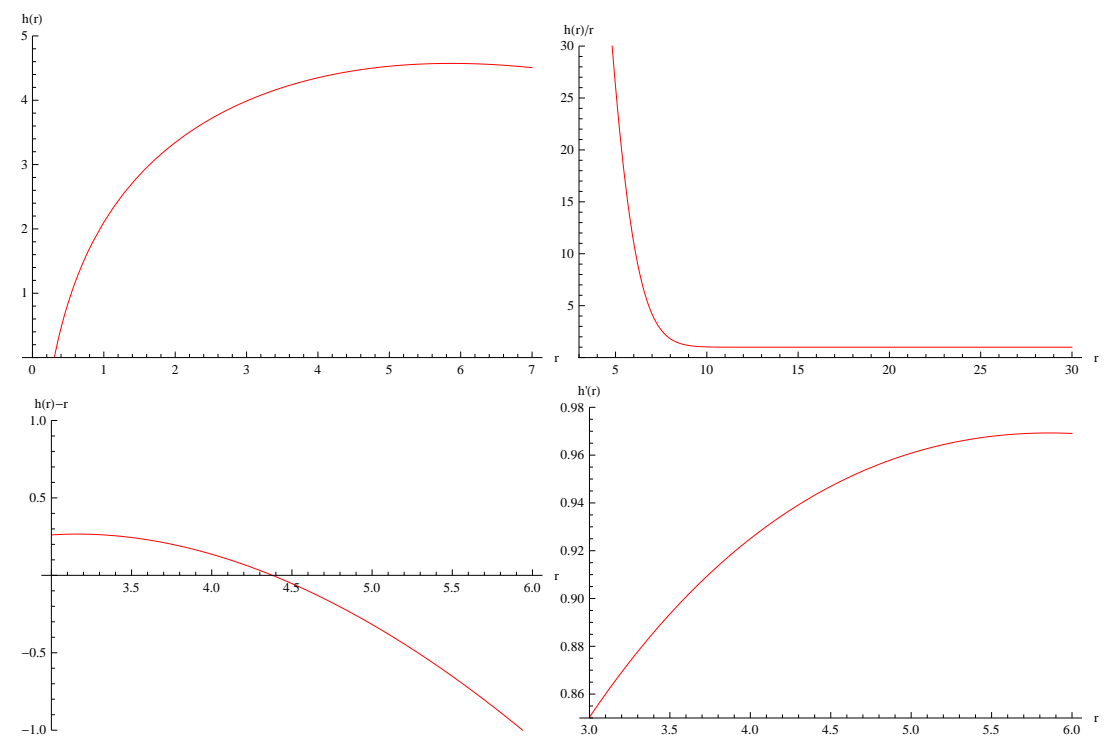

Figure 2: Plots of $h(r), \frac{h(r)}{r}, h(r)-r$ and $\frac{d h(r)}{d r}$ versus $r$ for $c_{5}=-0.35$, $c_{6}=0.1$ and $c_{7}=-0.25$.
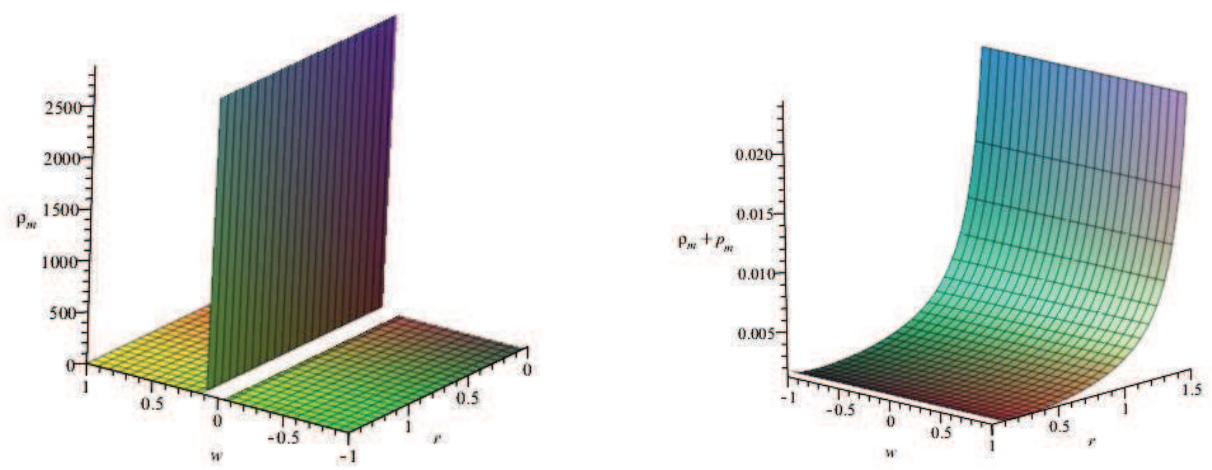

Figure 3: Plots of $\rho_{m}$ and $\rho_{m}+p_{m}$ versus $r$. 

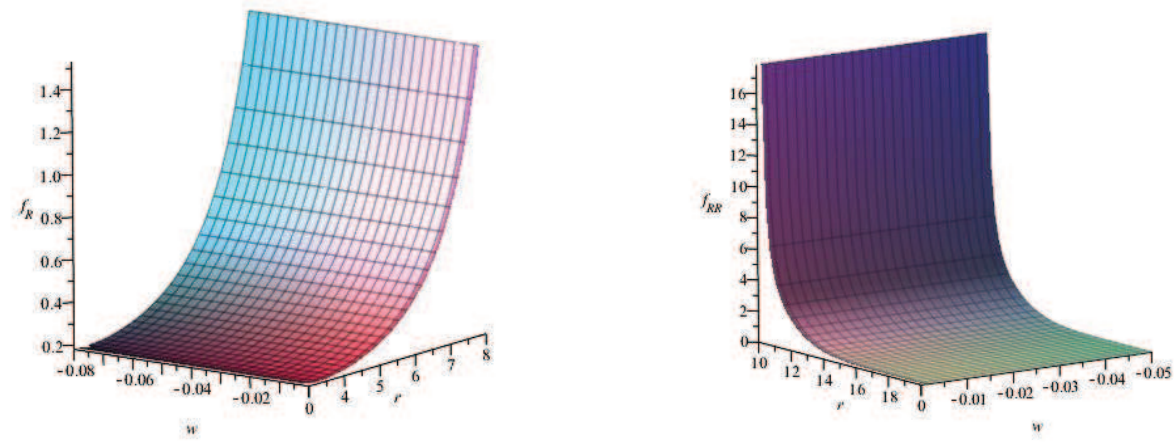

Figure 4: Stability conditions of $f(R)$ versus $r$ for $c_{2}=5, c_{4}=0.01, c_{5}=$ $-0.35, c_{6}=0.1, c_{7}=-0.25$ and $k=0.5$.

For this solution of $a(r)$ and $b(r)$, the generic function takes the form

$$
\begin{aligned}
f(R) & =-\frac{1}{2 c_{2}}\left[-(1+\omega) \rho_{0}\left(-\frac{k}{r}\right)^{-\frac{(1+3 \omega)}{2 \omega}}+2 \omega c_{2} \rho_{0}\left(-\frac{k}{r}\right)^{-\frac{(1+\omega)}{2 \omega}}-6 c_{4}\right. \\
& \left.\times \sqrt{c_{8} r^{4}(4 r+k)^{-4+c_{7}-\frac{k^{2} c_{6}}{8}}} e^{\frac{-\left(c_{6} r^{2}-\frac{c_{6} k r}{2}-\frac{k}{c_{8} r}\right)-c_{5}+k}{2}}\right]
\end{aligned}
$$

The corresponding shape function becomes

$$
h(r)=r\left(1-c_{8} r^{4}(4 r+k)^{-4+c_{7}-\frac{k^{2} c_{6}}{8}} e^{-\left(c_{6} r^{2}-\frac{c_{6} k r}{2}-\frac{k}{c_{8} r}\right)-c_{5}}\right) .
$$

Figure 4 shows that the model (47) follows the stability condition for $0<\omega<-0.08$ whereas Figure 5 represents the graphical behavior of the shape function. In upper face, the left plot preserves the positivity of $h(r)$ while the right plot ensures asymptotic flat geometry of WH. In lower face, the left plot detects WH throat at $r_{0}=5.878$ whereas the right plot indicates that $\frac{d h\left(r_{0}\right)}{d r}=0.1673<1$. For Eqs.(12) and (46), we obtain

$$
\rho_{e f f}+p_{e f f}=\frac{k}{r^{2}(r-h(r))}+\frac{r h^{\prime}(r)-h(r)}{r^{3}} .
$$

To investigate the presence of realistic traversable WH, we establish the graphical behavior of NEC and WEC corresponding to perfect fluid as well 

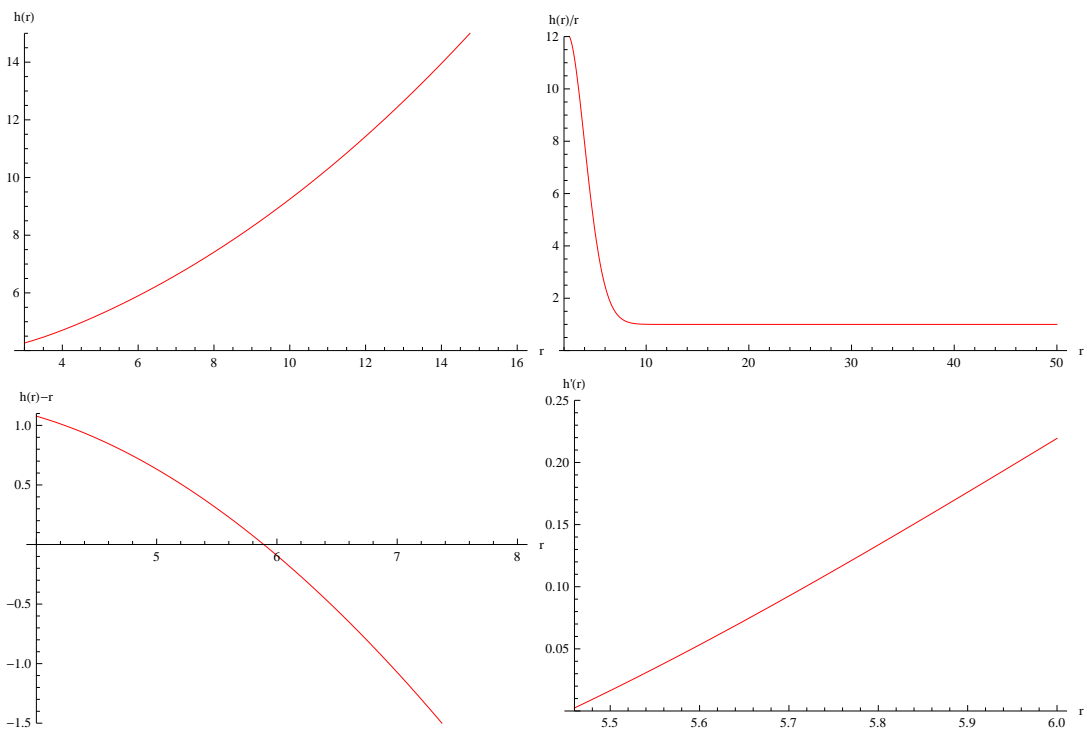

Figure 5: Plots of $h(r), \frac{h(r)}{r}, h(r)-r$ and $\frac{d h(r)}{d r}$ versus $r$ for $c_{5}=-4, c_{6}=0.1$, $c_{8}=-1$ and $k=0.25$.
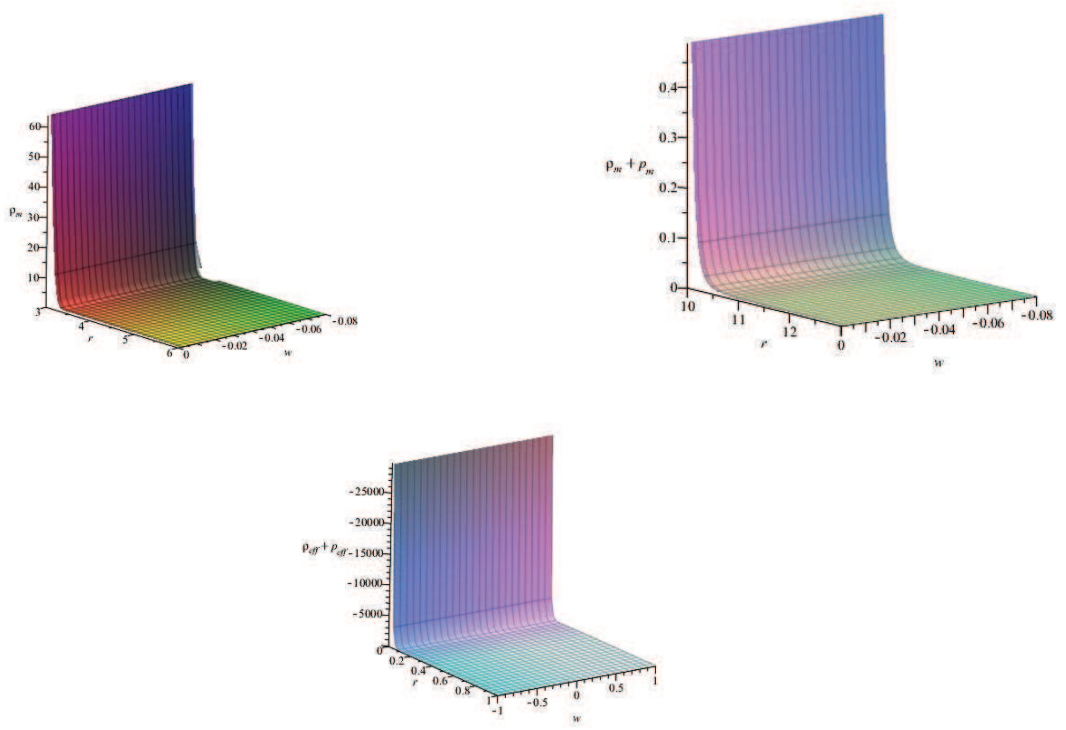

Figure 6: Plots of $\rho_{m}, \rho_{m}+p_{m}$ and $\rho_{e f f}+p_{\text {eff }}$ versus $r$. 
as NEC relative to effective energy-momentum tensor. Figure 6 indicates that $\rho_{m}+p_{m} \geq 0, \rho_{m} \geq 0$ and $\rho_{\text {eff }}+p_{\text {eff }}<0$ for $1<\omega<-1$. Thus, the physical existence of WH is assured in this case.

\subsection{Power-law $f(R)$ Model}

Here, we construct a WH solution with symmetry generator and corresponding conserved quantity for $f(R)$ power-law model, i.e., $f(R)=f_{0} R^{n}, n \neq$ 0,1 . For this purpose, we solve Eqs.(27)-(35) leading to

$$
\alpha=Y_{3}(a, r), \quad \gamma=Y_{1}(r), \quad \delta=Y_{2}(r, R) .
$$

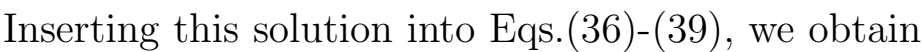

$$
Y_{1}(r)=0, \quad Y_{3}(a, r)=d_{2}, \quad Y_{2}(r, R)=d_{1} R, \quad \beta=2(n-1) d_{1}+d_{2}-2 \tau,{ }_{r},
$$

where $d_{1}$ and $d_{2}$ represent arbitrary constants. For these values, the coefficients of symmetry generator turn out to be

$$
\alpha=d_{2}, \quad \beta=2(n-1) d_{1}+d_{2}-2 \tau,_{r}, \quad \gamma=0, \quad \delta=d_{1} R .
$$

Substituting these coefficients in Eq.(40) and assuming $B=d_{0}$ and $\tau=\tau_{0}$, it follows that

$$
\begin{aligned}
b(r) & =\int \frac{8 d_{3} r^{2}+2 a^{\prime \prime} r^{2}+4 a^{\prime} r^{\prime}+a^{\prime 2} r^{2}-4 d_{4}}{r\left(4+a^{\prime} r\right)} d r \\
& -\ln \left[-d_{1}+4 \int \frac{e^{\int \frac{8 r^{2}+2 a^{\prime \prime} r^{2}+4 a^{\prime} r^{\prime}+a^{\prime 2} r^{2}-4}{r\left(4+a^{\prime} r\right)} d r}}{r\left(4+a^{\prime} r\right)} d r\right] .
\end{aligned}
$$

The resulting coefficients of symmetry generator verifies the system (27)-(39) for $d_{2}=-2(n-1) d_{1}$. Under this condition, the symmetry generator and associated first integral take the form

$$
\begin{aligned}
K & =\tau_{0} \frac{\partial}{\partial r}-2(n-1) d_{1} \frac{\partial}{\partial a}+d_{1} \frac{\partial}{\partial R} \\
\Sigma & =d_{0}-\tau_{0}\left[e^{\frac{a}{2}} e^{\frac{b}{2}} r^{2}\left(f-R f_{R}+\omega \rho_{0} a^{-\frac{(1+\omega)}{2 \omega}}+2 f_{R} r^{-2}\right)+\frac{e^{\frac{a}{2}} r^{2}}{e^{\frac{b}{2}}}\right. \\
& \left.\times\left\{f_{R}\left(2 r^{-2}+2 a^{\prime} r^{-1}\right)+f_{R R}\left(a^{\prime} R^{\prime}+4 R^{\prime} r^{-1}\right)\right\}\right]-2 d_{1}(1-n) e^{\frac{a-b}{2}}\left(R^{\prime} r^{2}\right. \\
& \left.\times f_{R R}+2 r f_{R}\right)-d_{1} R f_{R R} e^{\frac{a-b}{2}}\left(a^{\prime} r^{2}+4 r\right) .
\end{aligned}
$$

Now, we solve the integral (50) for constant and variable forms of red-shift function and study WH geometry via shape function. 


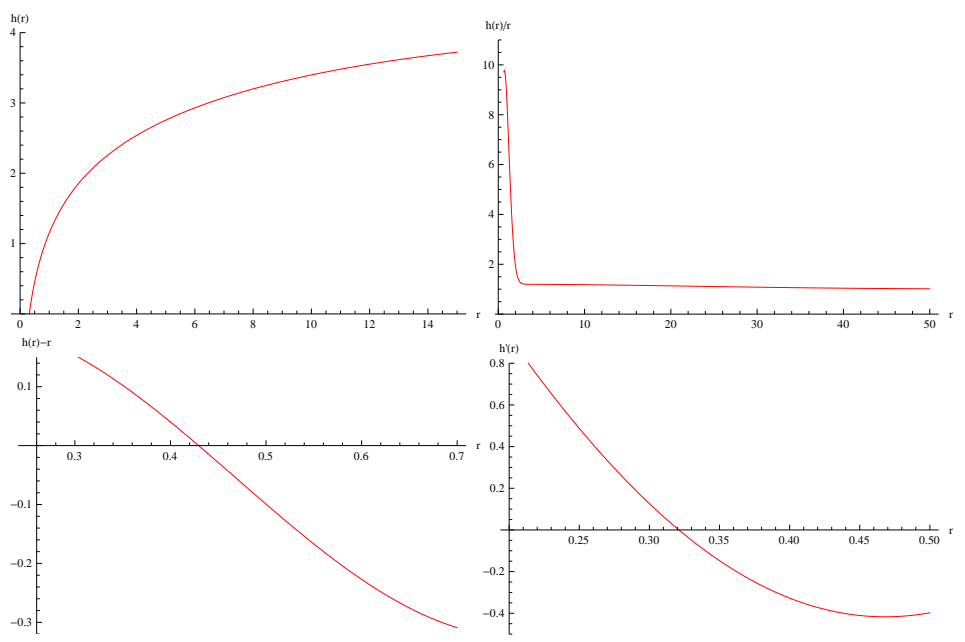

Figure 7: Plots of $h(r), \frac{h(r)}{r}, h(r)-r$ and $\frac{d h(r)}{d r}$ versus $r$ for $d_{2}=16, d_{3}=$ $1.001, d_{4}=-0.2$ and $n=\frac{1}{2}$.

Case I: $a(r)=k$

For constant red-shift function, the integral (50) reduces to

$$
b(r)=d_{3} r^{2}-d_{4} \ln r-\ln \left(\frac{-d_{1} r+e^{r^{2}}}{r}\right) .
$$

This satisfies Eq.(40) for $\omega=1, \frac{1}{3},-\frac{1}{3},-1$ and

$$
\rho_{0}=-\frac{f_{o} e^{\frac{3 \omega \ln d_{1}+4 n \omega \ln 2+\ln d_{1}}{2 \omega}}}{\omega d_{1}-(1+\omega)}, \quad \omega \neq 0 .
$$

In this case, the shape function yields

$$
h(r)=r\left[1-d_{4} r\left(\frac{-d_{1} r+e^{r^{2}}}{r}\right) e^{-d_{3} r^{2}}\right] .
$$

We analyze WH geometry via shape function for $n=\frac{1}{2}, 2$ and $n=4$. In upper face, the left and right plots of Figure $\mathbf{7}$ show that $h(r)$ remains positive and asymptotic flat for $n=\frac{1}{2}$. The lower left plot identifies WH throat at $r_{0}=5.101$ and right plot satisfies the condition, i.e., $h^{\prime}\left(r_{0}\right)=0.17<1$. In 


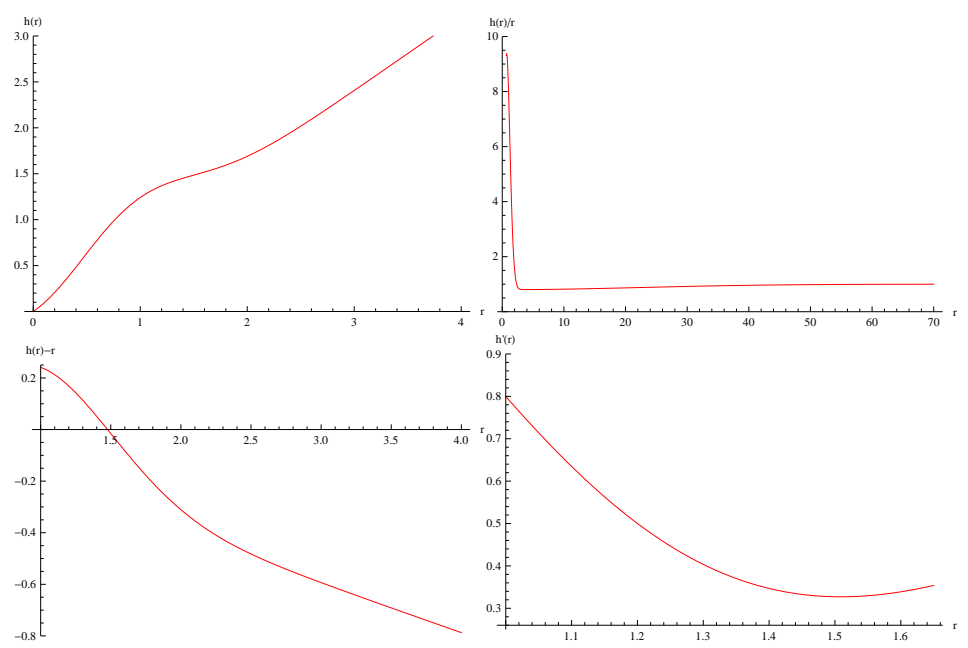

Figure 8: Plots of $h(r), \frac{h(r)}{r}, h(r)-r$ and $\frac{d h(r)}{d r}$ versus $r$ for $d_{2}=-200$, $d_{3}=1.001, d_{4}=0.2$ and $n=2$.

Figures $\mathbf{8}$ and $\mathbf{9}$, the shape function preserves its positivity condition and also admits asymptotic flat geometry for both $n=2$ and $n=4$. The WH throat is located at $r_{0}=0.23$ and $r_{0}=2.052$ for $n=2$ and $n=4$, respectively. The derivative condition is also satisfied at throat, i.e., $h^{\prime}\left(r_{0}\right)=0.89<1$ and $h^{\prime}\left(r_{0}\right)=-0.49<1$. The NEC relative to effective energy-momentum tensor verifies $\rho_{\text {eff }}+p_{\text {eff }}<0$ while Figure 10 identifies $\rho_{m} \geq 0$ and $\rho_{m}+p_{m} \geq 0$ for $n=0.5$. In case of $n=2$ and $n=4$, the energy density and pressure corresponding to perfect fluid evolve in the same way.

Case II: $a(r)=-k / r$

Here we consider red-shift function to be $r$-dependent and solve the integral (50) implying that

$$
\begin{aligned}
b(r) & =r^{2}-\frac{r d_{1}(1-n)}{2}+\frac{d_{1}^{2}(1-n)^{2} \ln \left(d_{1}(1-n)+4 r\right)}{8}+\left(d_{1}(1-n)\right)^{2} \\
& \times\left\{-\frac{1}{r d_{1}(1-n)}+\frac{4 \ln \left(d_{1}(1-n)+4 r\right)}{\left(d_{1}(1-n)\right)^{2}}-\frac{4 \ln r}{\left(d_{1}(1-n)\right)^{2}}\right\}-\ln ((1-n) \\
& \left.\times d_{1}+4 r\right)-\ln \left[4 \int \frac { 1 } { 4 r + d _ { 1 } ( 1 - n ) } \left(r^{-4}\left(d_{1}(1-n)+4 r\right)^{3+\frac{d_{1}^{2}(1-n)^{2}}{8}}\right.\right. \\
& \left.\left.\times e^{r^{2}-\frac{r d_{1}(1-n)}{2}+\frac{d_{1}(1-n)}{r}}\right) d r-d_{1}\right] .
\end{aligned}
$$




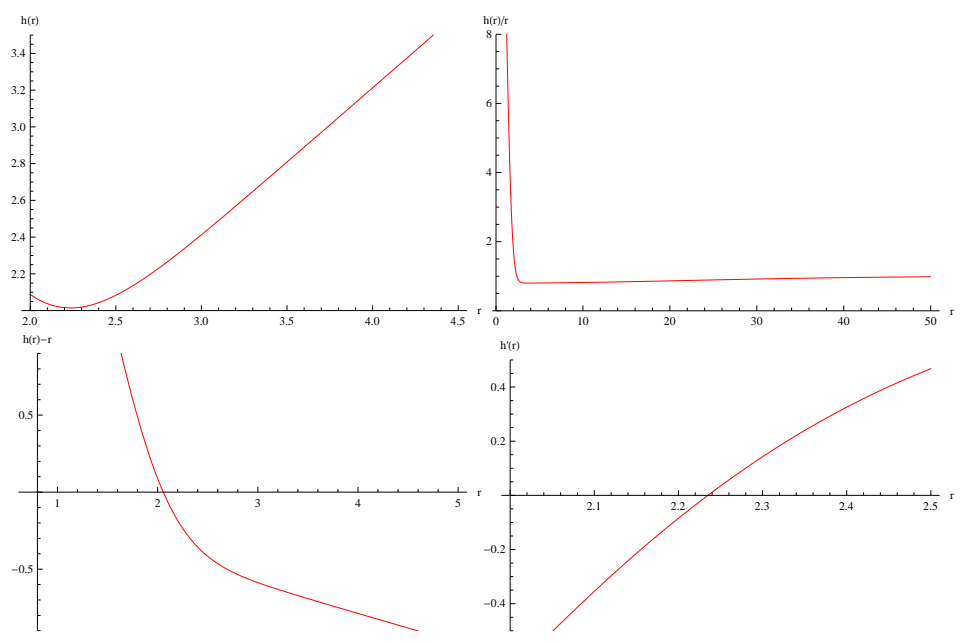

Figure 9: Plots of $h(r), \frac{h(r)}{r}, h(r)-r$ and $\frac{d h(r)}{d r}$ versus $r$ for $d_{2}=-200$, $d_{3}=1.001, d_{4}=0.2$ and $n=4$.
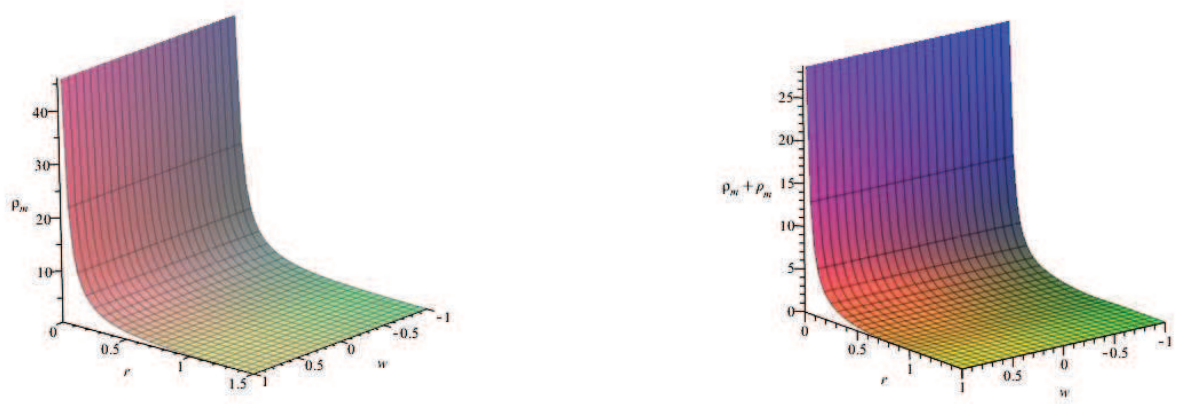

Figure 10: Plots of $\rho_{m}$ and $\rho_{m}+p_{m}$ versus $r$ for $n=0.5$. 
This solution satisfies Eq.(40) for $\omega=-1$. The shape function of WH takes the form

$$
\begin{aligned}
& \frac{h(r)}{r}=\left(1-r^{4}\left(d_{1}(1-n)+4 r\right)^{-3-\frac{d_{1}^{2}(1-n)^{2}}{8}} e^{-r^{2}+\frac{r d_{1}(1-n)}{2}-\frac{d_{1}(1-n)}{r}}\left[\int \left\{4 r+d_{1}\right.\right.\right. \\
& \left.\left.\times(1-n)\}^{-1}\left(r^{-4}\left(d_{1}(1-n)+4 r\right)^{3+\frac{d_{1}^{2}(1-n)^{2}}{8}} e^{r^{2}-\frac{r d_{1}(1-n)}{2}+\frac{d_{1}(1-n)}{r}}\right) d r-d_{1}\right]\right) .
\end{aligned}
$$

When red-shift function is not constant $\left(a^{\prime}(r) \neq 0\right)$, then the geometry of WH cannot be analyzed for $f(R)$ power-law model due to the complicated forms of $b(r)$ and $h(r)$.

\subsection{Exponential Model}

In this section, we consider another example of viable $f(R)$ model, i.e., exponential model to realize the existence of realistic traversable WH. The simplest version of this model is proposed as [30]

$$
f(R)=R-2 \Lambda\left(1-e^{-\frac{R}{R_{0}}}\right),
$$

where $\Lambda$ denotes cosmological constant while $R_{0}$ defines curvature parameter. If $R \gg R_{0}$, then the corresponding model recovers standard cosmological constant cold dark matter model. To formulate WH solution, we first solve the system of Eqs.(27)-(40) for the model (54) which leads to the following coefficients of symmetry generator and boundary term

$$
\begin{aligned}
\alpha & =0, \quad \beta=\frac{4 \Lambda \chi_{1}}{R_{0}}, \quad \gamma=0, \quad \delta=\chi_{1}\left(R_{0} e^{\frac{R}{R_{0}}}-2 \Lambda\right), \quad \tau=\tau_{0}, \\
B & =\frac{2 e^{\frac{a+b}{2}} \Lambda \chi_{1}}{R_{0}^{2}}\left[-\frac{2 r^{3} R_{0} \Lambda}{3}\left(1-e^{-\frac{R}{R_{0}}}-\frac{2 R}{R_{0}}\right)+\frac{r^{3} R_{0}\left(1-R R_{0}\right)}{3}+4 r\right. \\
& \left.\times\left(R_{0}-2 \Lambda e^{-\frac{R}{R_{0}}}\right)\right]+\chi_{2},
\end{aligned}
$$

where $\chi_{1}$ and $\chi_{2}$ represent arbitrary constants. These solutions satisfy the system for $\omega=\rho_{0}=-1$ and the following constraint

$$
e^{\frac{R}{R_{0}}} r^{2} R_{0}^{2}-2 r^{2} R_{0} \Lambda+4 r^{2} R \Lambda-24 \Lambda=0 .
$$

Now we determine the coefficient of radial component of the metric (5) using this constraint with Eq.(19) for both constant as well as variable forms of red-shift function and study WH geometry via shape function. 
Case I: $a(r)=k$

In this case, we obtain

$$
\begin{aligned}
e^{b(r)} & =-\left(4 \left(-2 R_{0} r^{2}+\left(R_{0} r^{2}+12 r^{4}\right) \exp \left((1 / 2)\left(12+R_{0} r^{2}\right) /\left(R_{0} r^{2}\right)\right)\right.\right. \\
& \left.\left.-48 r^{4} \chi_{4}+24\left(1-\chi_{4}\right)\right)\right)\left\{\left(r ^ { 2 } \left(\left(5 r^{4} R_{0}^{2}-2 r^{4} R_{0}-4 R_{0} r^{2}\right)\right.\right.\right. \\
& \times \exp \left((1 / 2)\left(12+R_{0} r^{2}\right)\left(R_{0} r^{2}\right)^{-1}\right)-6 r^{4} R_{0}^{2}+48 r^{2} \chi_{4}-48 r^{2} \\
& \left.\left.\left.-120 R_{0} r^{2} \chi_{4}+104 R_{0} r^{2}-96+96 \chi_{4}\right)\right)\right\}^{-1} .
\end{aligned}
$$

From this expression, we formulate shape function through $h(r)=r\left[1-e^{-b(r)}\right]$ and analyze the WH geometry graphically. In Figure 11, the upper face indicates that the shape function is positively increasing while the corresponding geometry is found to be asymptotically flat as $h(r) / r \rightarrow 0$ when $r \rightarrow \infty$. In the lower face, the left plot indicates that the $\mathrm{WH}$ throat exists at $r_{0}=0.05$ and also preserves the condition, i.e., $h(0.05)=0.05$ while the right plot shows that $h^{\prime}\left(r_{0}\right)=-0.007<1$. Since the red-shift function is constant therefore, the traversable nature of the constructed $\mathrm{WH}$ solution is preserved by the violation of effective NEC, i.e., $p_{e f f}+\rho_{e f f}<0$. Figure 12 evaluates the criteria for physically viable $\mathrm{WH}$ as $\rho_{m}>0$ and $p_{m}+\rho_{m}>0$.

Case II: $a(r)=-k / r$

Using Eqs.(19) and (155), it follows that

$$
\begin{aligned}
e^{b(r)} & =-\left(4 \left(24+48 k r^{2}-2 R_{0} r^{2}-4 k r^{4} R_{0}-12(r+4) k r^{2} \chi_{4}\right.\right. \\
& \left.-24 \chi_{4}\left(1+2 r^{4}\right)\right)+\left(2 k r^{4} R_{0}+3 r^{3} k+R_{0} r^{2}+12 r^{4}\right) \\
& \left.\times \exp \left((1 / 2)\left(12+R_{0} r^{2}\right) /\left(R_{0} r^{2}\right)\right)\right)\left\{r ^ { 2 } \left(\left(-2 r^{4} R_{0}+5 r^{4} R_{0}^{2}-4 R_{0} r^{2}\right)\right.\right. \\
& \times \exp \left((1 / 2)\left(12+R_{0} r^{2}\right) /\left(R_{0} r^{2}\right)\right)-\left(6 r^{2} R_{0}+104\right) R_{0} r^{2}-48\left(r^{2}+2\right) \\
& \left.-\left(120 R_{0} r^{2}-48 r^{2}+96\right) \chi_{4}\right\}^{-1} .
\end{aligned}
$$

Inserting the above expression in $h(r)=r\left[1-e^{-b(r)}\right]$, we construct WH solution relative to variable but finite red-shift function whose graphical interpretation is given in Figure 13. Both plots of the upper and lower panels indicate that the constructed $\mathrm{WH}$ follows asymptotic flat geometry whose throat is located at $r_{0}=0.01$ and $h^{\prime}(0.01)=-0.001<1$. In order to analyze the presence of repulsive gravitational effects at throat, we study the behavior of effective NEC in Figure 14 which ensures that the sum of $p_{\text {eff }}$ and $\rho_{\text {eff }}$ remains negative. Thus, the constructed $\mathrm{WH}$ is found to be traversable. Both plots of Figure $\mathbf{1 5}$ shows that the WH is physically viable as NEC and WEC corresponding to ordinary matter are preserved. 

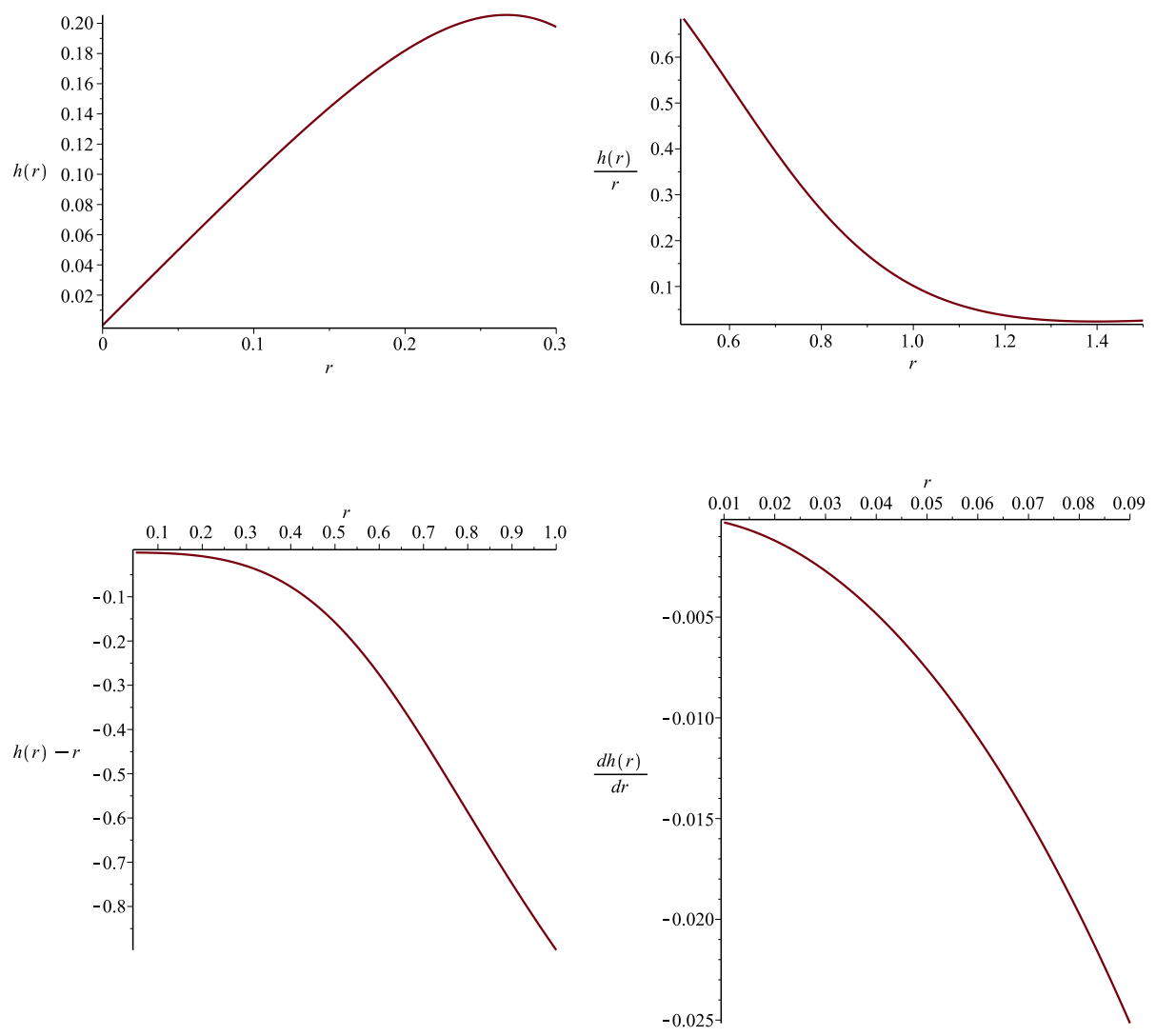

Figure 11: Plots of $h(r), \frac{h(r)}{r}, h(r)-r$ and $\frac{d h(r)}{d r}$ versus $r$ for $\chi_{4}=-200$, $R_{0}=-0.95=\Lambda$ and $k=0.005$. 

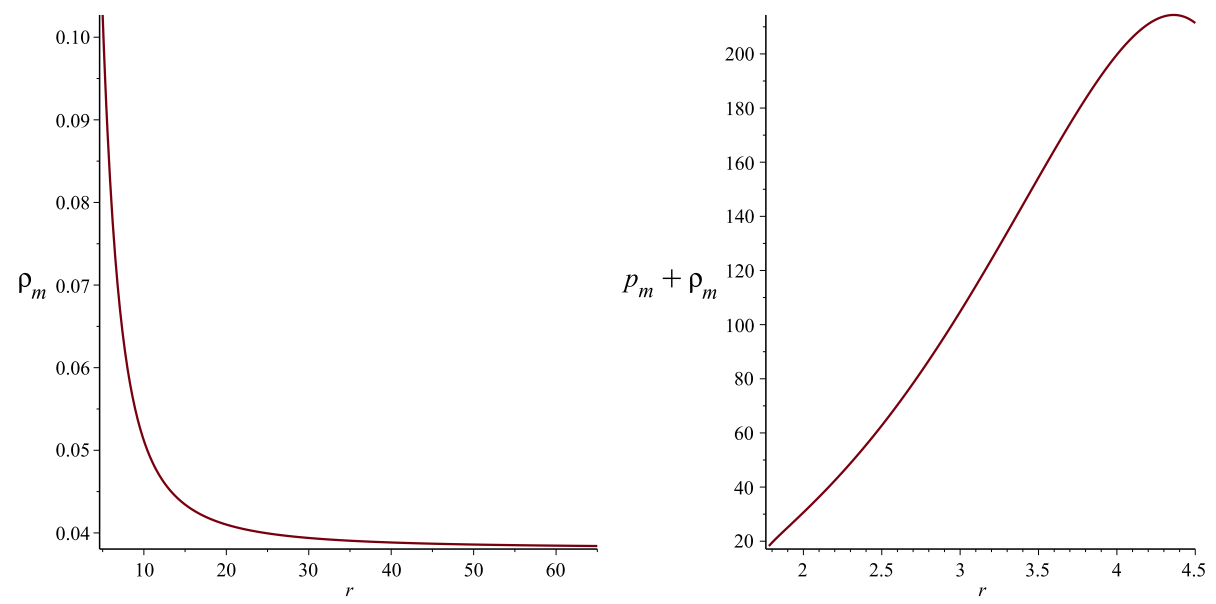

Figure 12: Plots of $\rho_{m}$ and $\rho_{m}+p_{m}$ versus $r$.

\section{$5 \quad$ Stability Analysis}

Here we discuss the stability of WH solutions relative to both constant as well as variable red-shift function via Tolman-Oppenheimer-Volkov (TOV) equation. For isotropic fluid distribution, the radial component of Bianchi identity $\left(\nabla_{\mu} T^{\mu \nu}=0\right)$ defines TOV equation as

$$
\frac{d p_{m}}{d r}+\frac{a^{\prime}(r)}{2}\left(p_{m}+\rho_{m}\right)=0
$$

The conservation of energy-momentum tensor relative to high order curvature terms leads to

$$
T_{11}^{\prime(c)}+\frac{a^{\prime}}{2}\left(T_{00}^{(c)}+T_{11}^{(c)}\right)-\frac{M^{\prime}}{M}\left(f_{R}^{\prime \prime}-\frac{f_{R}^{\prime}}{e^{b(r)}}\left\{\frac{b^{\prime}}{2}+\frac{M^{\prime}}{2 M}\right\}\right)=0 .
$$

Combining Eq.(57) and (58), it follows that

$$
p_{(e f f)}^{\prime}+\frac{a^{\prime}(r)}{2}\left(p_{e f f}+\rho_{e f f}\right)-\frac{M^{\prime}}{M}\left(f_{R}^{\prime \prime}-\frac{f_{R}^{\prime}}{e^{b(r)}}\left\{\frac{b^{\prime}}{2}+\frac{M^{\prime}}{2 M}\right\}\right)=0,
$$

where $p_{\text {eff }}=p_{m}+T_{11}^{(c)}$ and $\rho_{\text {eff }}=\rho_{m}+T_{00}^{(c)}$. This equation determines the fate of the WH as it can be expressed as a combination of hydrostatic $\mathcal{F}_{h}$ and gravitational force $\mathcal{F}_{g}$. Using Eq.(59), these forces take the following form

$$
\mathcal{F}_{h}=p_{(e f f)}^{\prime}=\frac{d}{d r}\left(p_{m}+T_{11}^{(c)}\right)
$$



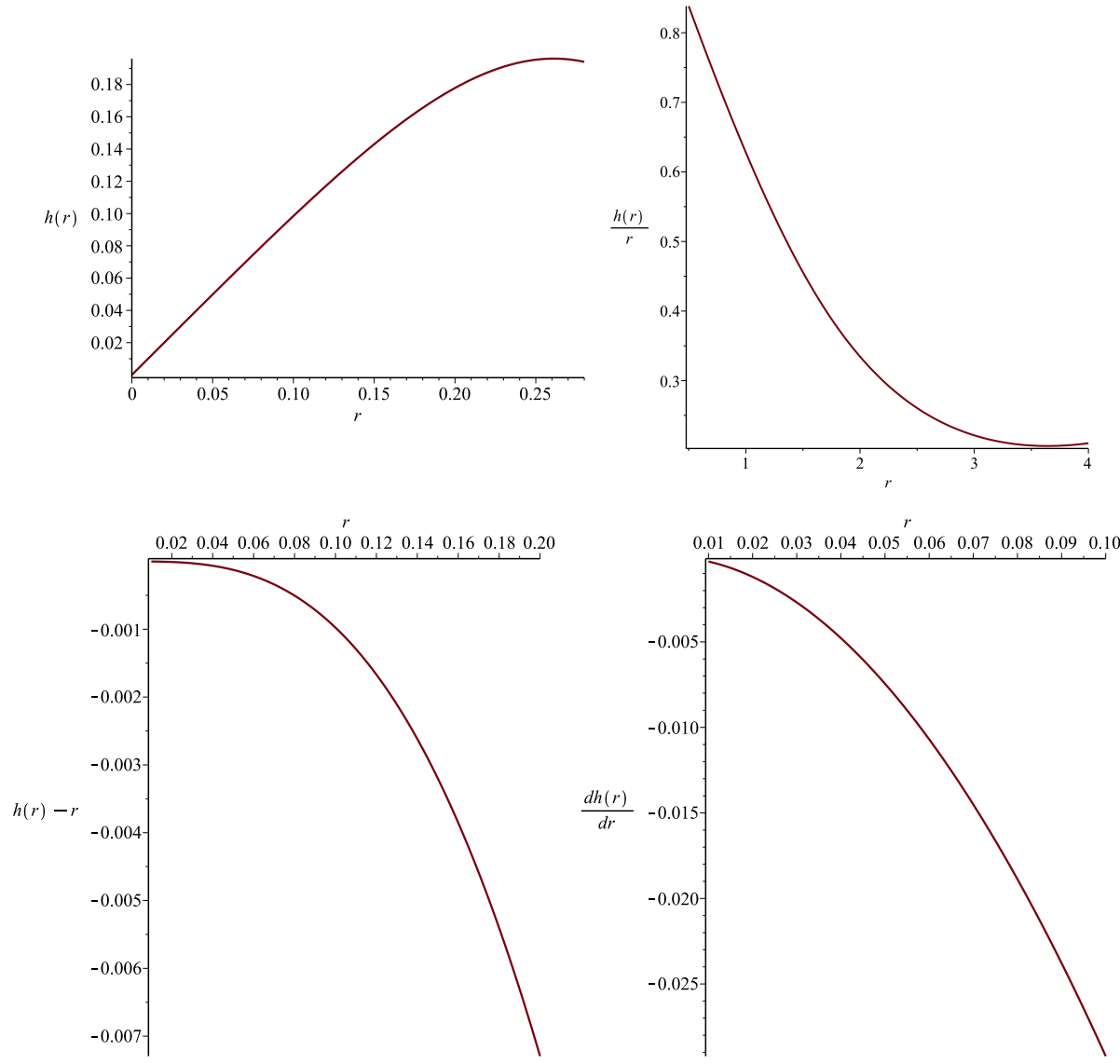

Figure 13: Plots of $h(r), \frac{h(r)}{r}, h(r)-r$ and $\frac{d h(r)}{d r}$ versus $r$ for $\chi_{4}=-0.20$, $R_{0}=-0.95=\Lambda$ and $k=2$. 


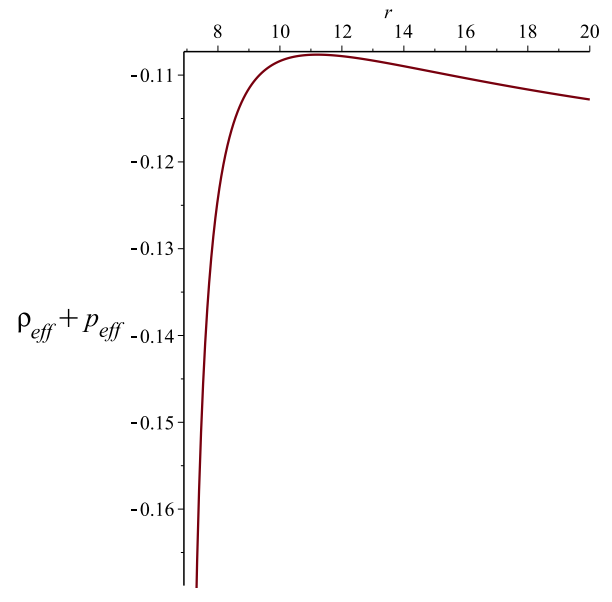

Figure 14: Evolution of $\rho_{e f f}+p_{\text {eff }}$ versus $r$.
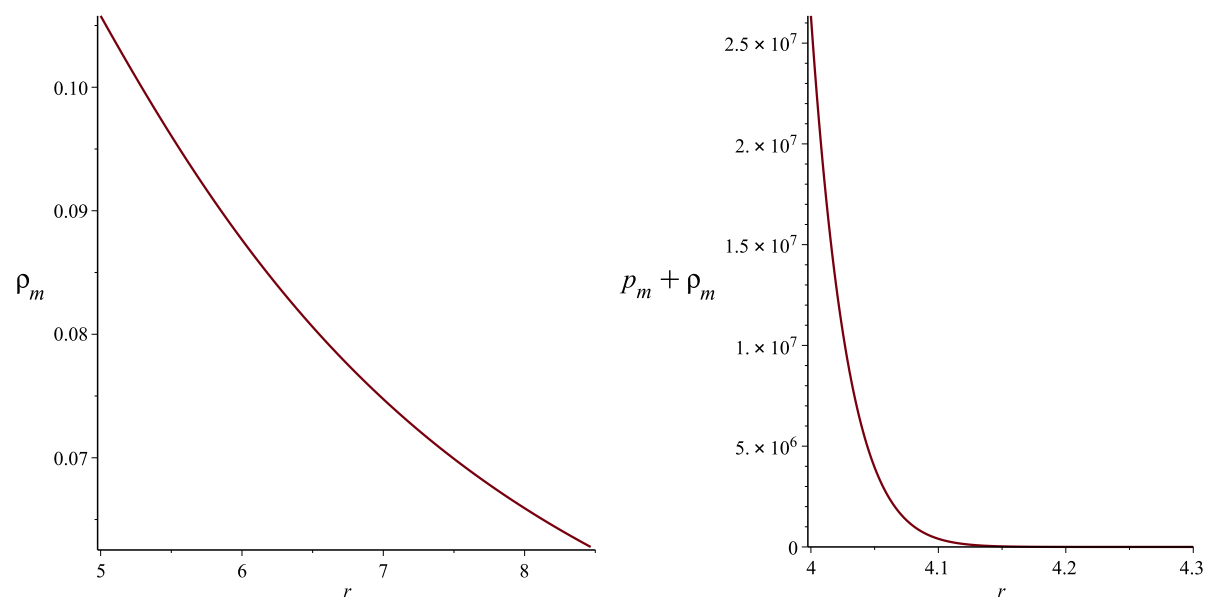

Figure 15: Plots of $\rho_{m}$ and $\rho_{m}+p_{m}$ versus $r$. 

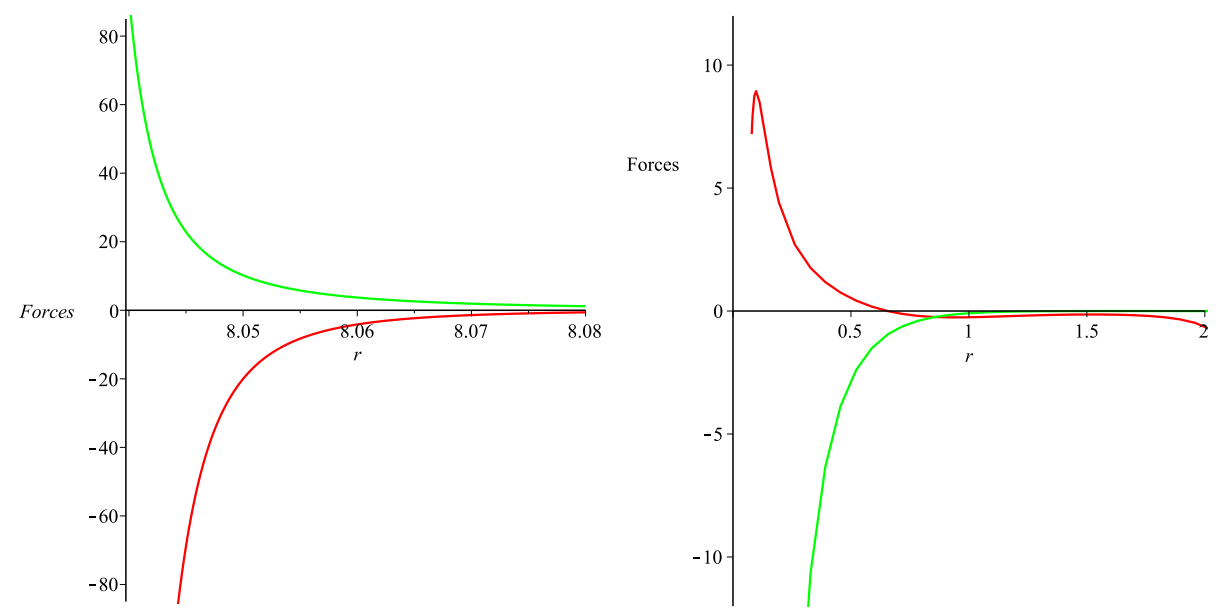

Figure 16: Plots of $\mathcal{F}_{g}$ (green) and $\mathcal{F}_{h}$ (red) versus $r$ for $a(r)=k$ (left) and $a(r)=-k / r$ (right) for $c_{2}=5, c_{4}=0.01, c_{5}=-0.35, c_{6}=0.1, c_{7}=-0.25$, $\rho_{0}=-0.01$ and $k=0.5$.

$$
\mathcal{F}_{g}=\frac{\mathcal{M}_{e f f} e^{\frac{a-b}{2}}}{r^{2}}\left(p_{e f f}+\rho_{e f f}\right)-\frac{M^{\prime}}{M}\left(f_{R}^{\prime \prime}-\frac{f_{R}^{\prime}}{e^{b(r)}}\left\{\frac{b^{\prime}}{2}+\frac{M^{\prime}}{2 M}\right\}\right)
$$

where $\mathcal{M}_{\text {eff }}=\frac{a^{\prime} r^{2} e^{\frac{b-a}{2}}}{2}$ denotes effective gravitational mass. The null effect $\left(\mathcal{F}_{h}+\mathcal{F}_{g}=0\right)$ of these dynamical forces leads to stable state of a $\mathrm{WH}$.

In Figures 16-18, we analyze the stability of WH solutions constructed with the help of a new $f(R)$ model as well as power-law and exponential forms of generic function $f(R)$. In Figure 16, the left plot represents the stability of WH solution (44) relative to constant red-shift function and $f(R)$ model (45). The effect of gravitational and hydrostatic forces appear to be the same but in opposite directions canceling each other effect. Thus, the considered WH is found to be stable due to null effect of these forces. For variable redshift function, the equilibrium state of WH solution (48) is analyzed in the right plot of Figure 16. Initially, the WH geometry seems to be unstable but gradually it attains an equilibrium state due to equal but opposite effect of hydrostatic and gravitational forces. Figure $\mathbf{1 7}$ determines the existence of stable WH for $n=0.5, n=2$ and $n=4$ with constant red-shift function. For $n=0.5$ and $n=0.4$, the system remains unstable as $\mathcal{F}_{g}+\mathcal{F}_{h} \neq 0$ whereas the constructed WH attains a stable state for $n=2$. In Figure 18, the WH solutions gradually attain equilibrium state corresponding to both forms of 


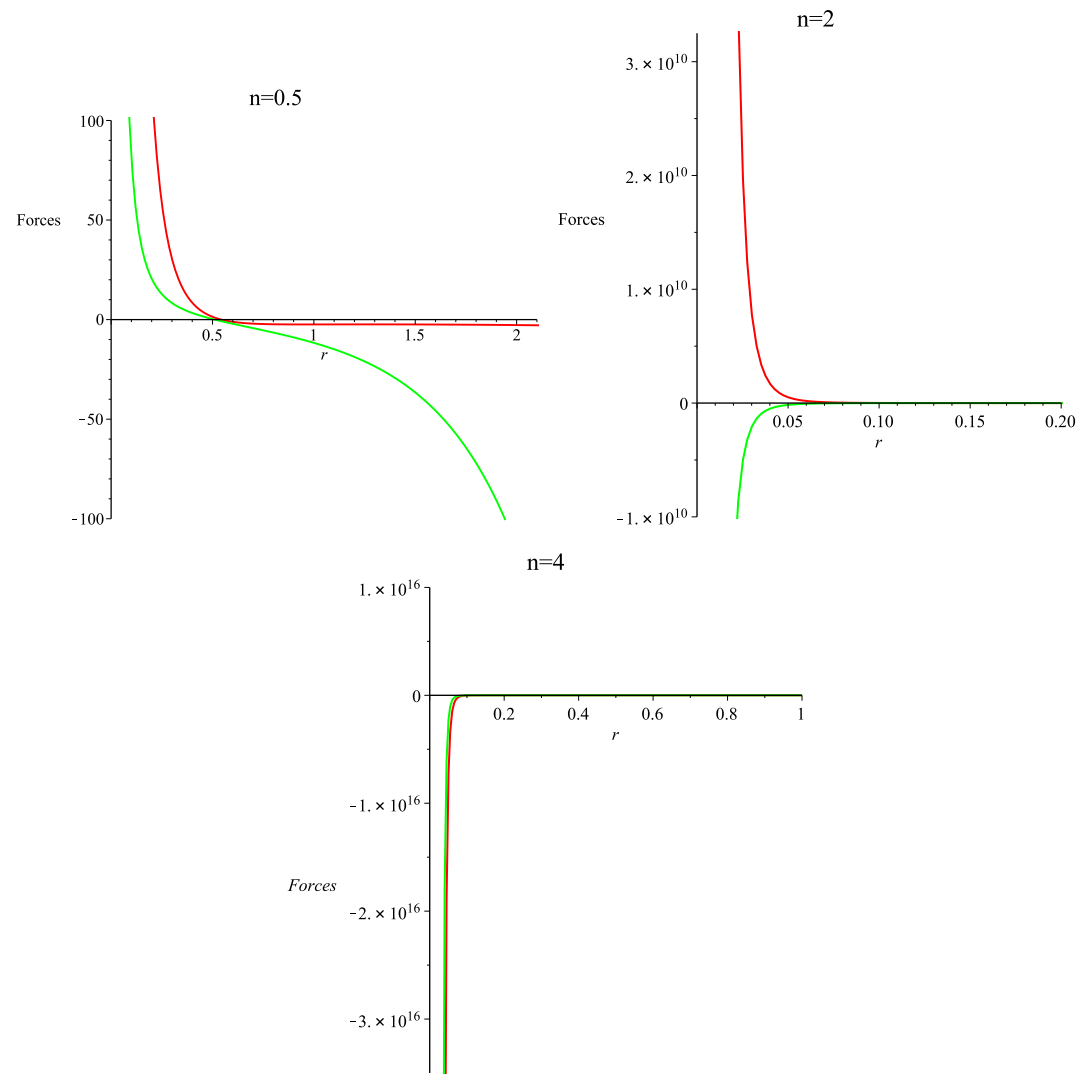

Figure 17: Plots of $\mathcal{F}_{g}$ (green) and $\mathcal{F}_{h}$ (red) versus $r$ for $a(r)=k, d_{2}=-2.2$, $d_{3}=1.001, d_{4}=0.05, f_{0}=1$ and $\mathcal{M}_{\text {eff }}=2$. 

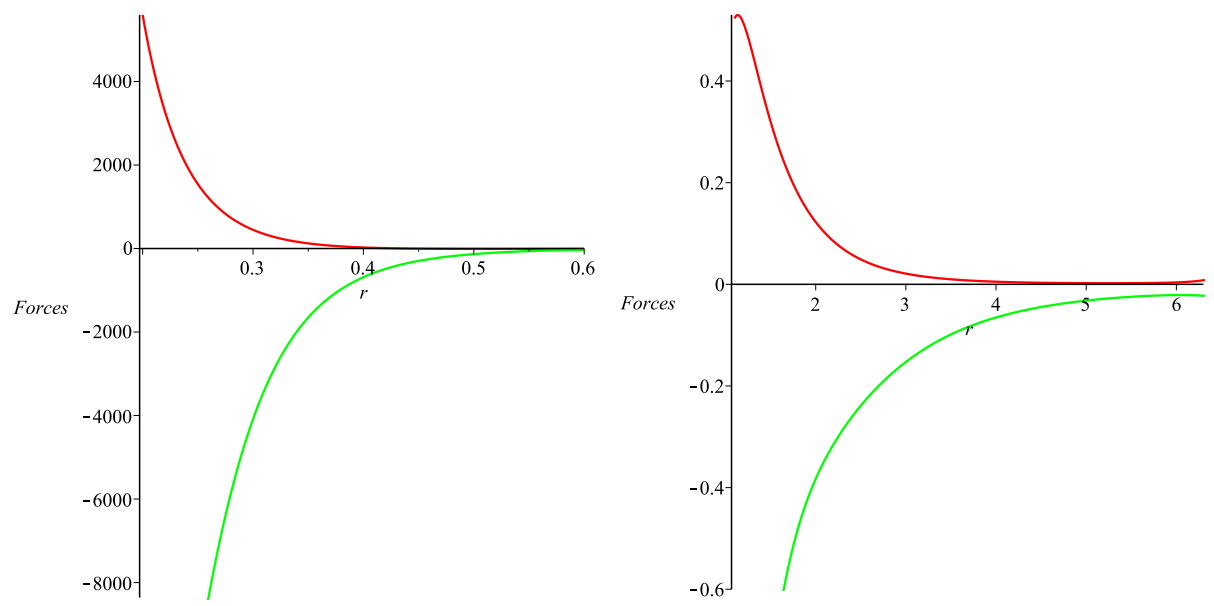

Figure 18: Plots of $\mathcal{F}_{g}$ (green) and $\mathcal{F}_{h}$ (red) versus $r$ for $a(r)=k$ (left), $k=0.005, \mathcal{M}_{\text {eff }}=-2$ and $a(r)=-k / r$ (right), $\chi_{4}=-0.2, R_{0}=-0.95$, $k=2$ and $\mathcal{M}_{\text {eff }}=2$.

red-shift function.

\section{Final Remarks}

In general relativity, the physical existence of a static traversable WH demands the violation of NEC by the energy-momentum tensor. This violation confirms the presence of exotic matter which would be minimized to have a physically viable WH. In case of $f(R)$ gravity, the energy-momentum tensor threading WH satisfies NEC and WEC whereas the existence of exotic matter is assured by the effective energy-momentum tensor which violates NEC. In this paper, we have discussed the presence of static traversable WH via Noether symmetry approach in $f(R)$ gravity. For this purpose, we have considered perfect fluid distribution and studied possible existence of realistic WH solutions for generic as well as $f(R)$ power-law model. We have solved over-determined system by invariance condition and found symmetry generator, associated conserved quantity, exact solution of $f(R)$ and $b(r)$ for static spherically symmetric metric. For these solutions, we have studied WH geometry and also investigated stable state of WH solutions via modified TOV equation for the red-shift function when $a(r)=k,-k / r$.

In case of constant red-shift function, we have obtained viable $f(R)$ model 
and the shape function satisfies all the properties, i.e., $h(r)>0$, WH geometry is found to be asymptotic flat and $\frac{d h(r)}{d r}<1$ at $r=r_{0}$. The violation of NEC (using effective energy-momentum tensor) assures the presence of repulsive nature of gravity while existence of ordinary matter is supported by verification of NEC and WEC relative to perfect fluid. When $a^{\prime} \neq 0$, the $f(R)$ model preserves stability conditions for $0<\omega<-0.08$ and the shape function has preserved all conditions of traversable WH while $\rho_{\text {eff }}+p_{\text {eff }}<0$, $\rho_{m}+p_{m} \geq 0$ and $\rho_{m} \geq 0$ minimizing the presence of exotic matter due to the presence of repulsive gravity. These energy bounds confirm the presence of a realistic WH solution threaded by $T_{\mu \nu}^{(m)}$. Consequently, we have found a physically viable WH solution for $a^{\prime} \neq 0$. For both forms of red-shift function, the constructed WH solutions attain an equilibrium state as $\mathcal{F}_{g}+\mathcal{F}_{h}=0$.

We have also formulated symmetry generator, corresponding first integral and WH solutions for $f(R)$ power-law model. When $a^{\prime}(r)=0$, we have established graphical analysis of traversable WH conditions for $n=1 / 2, n=2$ and $n=4$. In this case, the shape function is found to preserve all conditions and $\rho_{\text {eff }}+p_{\text {eff }}<0$ assures the violation of NEC identifying the existence of exotic matter at throat. The consistent behavior of $\rho_{m} \geq 0$ and $\rho_{m}+p_{m} \geq$ indicate that the constructed traversable WH is supported by ordinary matter. The stability analysis of these realistic traversable WHs identifies that the WH geometry would be stable only for $n=2$. For $a^{\prime} \neq 0$, we have found a complicated form of the shape function. For exponential $f(R)$ model, the WH geometry is discussed near the throat. The shape of $\mathrm{WH}$ is found to be asymptotically flat for both constant as well as variable forms of the redshift function. The violation of effective NEC and verification of NEC as well as WEC of ordinary matter assure the presence of realistic traversable WH solutions. The total effect of gravitational and hydrostatic forces identifies equilibrium state of WHs in both cases.

The WH solutions are found in $f(R)$ gravity which is equivalent to BransDicke theory under a particular conformal transformation. Coule [31] established static unrealistic WH solutions in Einstein frame of $f(R)$ theory. Nandi et al. [32] examined the possibility of static WH solutions in the background of both Jordan and Einstein frames of Brans-Dicke theory. They found that the non-traversable WH exists in the former frame whereas in the latter frame, WH solutions do not exist at all unless energy conditions are violated by hand. Furey and de Benedictis [33] discussed geometry of the WH solutions near the throat while Bronnikov and Starobinsky [34] claimed that 
the existence of throat can be preserved under a conformal transformation. In general, the back transformation from Jordan to Einstein frames does not assure to get physical solutions. It has been even widely demonstrated that passing from one frame to the other can completely change the physical meaning as well as the stability of the solutions [35]. Bahamonde et al. [36] observed the presence of big-rip (type I) singularity in the Einstein frame of $f(R)$ gravity while along back mapping, the universe evolution is found to be singularity free.

In this paper, we have explored the existence of realistic and stable traversable WH solutions in the Jordan frame representation of $f(R)$ theory. It is worth mentioning here that the WH geometry is discussed at the throat in case of standard power-law and constructed $f(R)$ models whereas in case of exponential model, we have analyzed the WH geometry near the throat. The presence of repulsive gravity due to higher order curvature terms leads to traversable WHs while the existence of ordinary matter confirms the realistic nature of these traversable WH solutions in each case. For $f(R)$ power-law model, the WH solutions are stable only for $n=2$ while stability is preserved for both exponential as well as constructed $f(R)$ models. It would be interesting to analyze the presence of these configurations in the Einstein frame where contribution of scalar field may enhance the traversable nature as it introduces anti-gravitational effects. On the other hand, the back mapping of these frames may or may not ensure the presence of stable as well as realistic traversable wormholes.

\section{Acknowledgment}

This work has been supported by the Pakistan Academy of Sciences Project.

\section{References}

[1] Sharif M. and Shamir, M.F.: Class. Quantum Grav. 26(2009)235020; Gen. Relativ. Gravit. 42(2010)2643.

[2] Gutiérrez-Piñeres, A.C. and López-Monsalvo, C.S.: Phys. Lett. B 718(2013)1493.

[3] Sharif, M. and Zubair, M.: Astrophys. Space Sci. 349(2014)457. 
[4] Gao, C. and Shen, Y.G.: Gen. Relativ. Gravit. 48(2016)131.

[5] Capozziello, S., Stabile, A. and Troisi, A.: Class. Quantum Grav. 24(2007)2153.

[6] Capozziello, S., Stabile, A. and Troisi, A.: Class. Quantum Grav. 25(2008)085004; ibid. 27(2010)165008.

[7] Vakili, B.: Phys. Lett. B 16(2008)664.

[8] Momeni, D., Myrzakulov, R. and Güdekli, E.: Int. J. Geom. Methods Mod. Phys. 12(2015)1550101.

[9] Sharif, M. and Nawazish, I.: J. Exp. Theor. Phys. 120(2014)49; Sharif, M. and Shafique, I.: Phys. Rev. D 90(2014)084033; Sharif, M. and Fatima, H.I.: J. Exp. Theor. Phys. 122(2016)104; Sharif, M. and Nawazish, I.: Gen. Relativ. Gravit. 49(2017)76; Eur. Phys. J. C 77(2017)198; Mod. Phys. Lett. A 32(2017)1750136.

[10] Kashargin, P.E. and Sushkov, S.V.: Gravit. Cosmol. 14(2008)80; Eiroa, E.F. and Simeone, C.: Phys. Rev. D 82(2010)084039.

[11] Lobo, F.S.N. and Oliveira, M.A.: Phys. Rev. D 80(2009)104012.

[12] Jamil, M. et al.: J. Kor. Phys. Soc. 65(2014)917.

[13] Bahamonde, S. et al.: Phys. Rev. D 94(2016)044041.

[14] Mazharimousavi, S.H. and Halilsoy, M.: Mod. Phys. Lett. A 31(2016)1650203.

[15] Sharif, M. and Fatima, H.I.: Gen. Relativ. Gravit. 48(2016)148; Astrophys. Space Sci. 361(2016)127.

[16] Bahamonde, S. et al.: Phys. Rev. D 94(2016)084042.

[17] Morris, M.S. and Thorne, K.S.: Am. J. Phys. 56(1988)395.

[18] Ellis, G.F.R., Maartens, R. and MacCallum, M.A.H.: Relativistic Cosmology (Cambridge University Press, 2012).

[19] Schutz Jr, B.F.: Phys. Rev. D 2(1970)2762. 
[20] Carroll, S.M.: Spacetime and Geometry: An Introduction to General Relativity (Addison Wesley, 2004).

[21] Santos, J. et al.: Phys. Rev. D 76(2007)083513.

[22] Capozziello, S. et al.: Phys. Lett. B, 693(2010)198.

[23] Capozziello, S., De Laurentis, M. and Odintsov, S.D.: Eur. Phys. J. C 72(2012)2068.

[24] Hanc, J., Tuleja, S. and Hancova, M.: Am. J. Phys. 72(2004)428.

[25] Kar, S. and Sahdev, D.: Phys. Rev. D 52(1995)2030.

[26] Capozziello, S.: Int. J. Mod. Phys. D 11(2002)483; Nojiri, S. and Odintsov, S.D.: arXiv:hep-th/0308176.

[27] Dolgov, A.D. and Kawasaki, M.: Phys. Lett. B 573(2003)1.

[28] Nojiri, S. and Odintsov, S.D.: arXiv:hep-th/0307288.

[29] Faraoni, V: Phys. Rev. D 74(2006)104017.

[30] Cognola, G, et al. Phys. Rev. D 77(2008)046009.

[31] Coule, D.H.: Class. Quantum Grav. 10(1993)L25.

[32] Nandi, K.K. et al.: Phys. Rev. D 57(1998)823.

[33] Furey, N. and DeBenedictis, A.: Class. Quantum Grav. 22(2006)313.

[34] Bronnikov, K.A. and Starobinsky, A.A.: J Exp. Theo. Phys. Lett. 85(2007)1.

[35] Gorini, V. et al.: Phys. Rev. D 72(2005)103518.

[36] Bahamonde et al.: Ann. Phys. 373(2016)96. 